\title{
A Sustainable Slit Jet FTIR Spectrometer for Hydrate Complexes and Beyond
}

\author{
Hannes C. Gottschalk (D), Taija L. Fischer (D), Volker Meyer, Reinhard Hildebrandt, Ulrich Schmitt \\ and Martin A. Suhm *(D)
}

check for updates

Citation: Gottschalk, H.C.; Fischer T.L.; Meyer, V.; Hildebrandt, R.; Schmitt, U.; Suhm, M.A. A Sustainable Slit Jet FTIR Spectrometer for Hydrate Complexes and Beyond. Instruments 2021, 5, 12. https:// doi.org/10.3390/instruments5010012

Academic Editor: Antonio Ereditato

Received: 16 February 2021

Accepted: 10 March 2021

Published: 17 March 2021

Publisher's Note: MDPI stays neutra with regard to jurisdictional claims in published maps and institutional affiliations.

Copyright: (c) 2021 by the authors. Licensee MDPI, Basel, Switzerland. This article is an open access article distributed under the terms and conditions of the Creative Commons Attribution (CC BY) license (https:// creativecommons.org/licenses/by/ $4.0 /)$.
Institut für Physikalische Chemie, Georg-August-Universität Göttingen, Tammannstr. 6, 37077 Göttingen, Germany; hgottsc@gwdg.de (H.C.G.); tfische1@gwdg.de (T.L.F.); vhme@web.de (V.M.); rhildeb1@gwdg.de (R.H.); uschmit@gwdg.de (U.S.)

* Correspondence: msuhm@gwdg.de; Tel.: +49-551-3933112

\begin{abstract}
Fourier transform infrared (FTIR) absorption spectroscopy of cold molecules and clusters in supersonic slit jet expansions complements and extends more sensitive action spectroscopy techniques and provides important reference data for the latter. We describe how its major drawback, large substance and carrier gas consumption, can be alleviated by one to two orders of magnitude via direct and continuous recycling of the gas mixture. This is achieved by a combination of dry rotary lobe and screw pump compression. The signal-to-noise ratio is boosted by the established buffered giant gas pulse technique with full interferogram synchronization. The buildup of water impurities typically limits the recycling gain, but is turned into a feature for the study of hydrate complexes of volatile molecules. Continuous operation with a single gas filling over several days becomes practical and useful. Decadic absorbances in the low ppm range are detectable and the mid infrared range can be recorded simultaneously with the near infrared. The less straightforward hydration number assignment of spectral features in direct absorption spectroscopy is supported by a gradual water buildup at a rate of less than $0.5 \mathrm{mg} / \mathrm{h}$. A recent reassignment proposal for the water dimer $\mathrm{OH}$ stretching spectrum is refuted and vibrational spectra of vacuum-isolated ${ }^{18} \mathrm{O}$-water clusters are presented for the first time. Methanol docking on asymmetric ketones is used to illustrate the advantages and limitations of the recycling concept. Previous assignments of the hydrate complex of 1-phenylethanol are confirmed. Additional features of the setup await testing and refinement, but the recycling technique already substantially widens the applicability of direct absorption spectroscopy of neutral molecular clusters. It may be attractive for other high-throughput jet spectrometers.
\end{abstract}

Keywords: FTIR spectroscopy; supersonic jet expansion; molecular clusters; gas recycling; water dimer; hydrate complex

\section{Introduction}

The vibrational spectroscopy of cold molecules and molecular clusters contributes importantly to the understanding of (inter-)molecular forces and dynamics [1-4]. Supersonic jet expansions are still the method of choice for system preparation. Because there is always a distribution of neutral cluster sizes, size selective action spectroscopy techniques are advantageous [5-11]. They exploit the molecular effects of photon absorption such as ionization, fluorescence or dissociation, rather than directly detecting the loss of photons. However, these action techniques either require major instrumental effort or are else restricted to particular compound classes to avoid fragmentation and other distortive issues. Claims of universal action spectroscopy techniques based on ionization are frequently made [12-17], but do not always withstand closer scrutiny. Their molecular action nature can lead to expected and sometimes unexpected distortions from a linear and fragmentation-free response to the photon absorption event. Therefore, direct infrared absorption-despite its intrinsically lower sensitivity-still occupies an important niche in 
the field. This may appear surprising, given the enormous background load of thermal photons in the infrared, but if frequency-specific photon losses on the order of a few ppm can be reliably detected, direct absorption can indeed compete with action spectroscopy of neutral clusters $[18,19]$. Besides providing an essential reference to judge new action spectroscopies, it can be quite productive by itself, if small clusters do not spectrally overlap with monomers and nearby cluster sizes. This is usually the case for hydrogen-bonded dimers and trimers in the hydride stretching region, but also in other favourable constellations. It is well known that supersonic expansions based on slit orifices [20-22] produce clusters more easily due to a larger number of collisions and they offer a scalable column density for signal enhancement $[18,19]$. When coupled to interferometric FTIR spectroscopy and standard incoherent light sources, slit expansions provide a universal perspective on cold vibrations from 200 to $8000 \mathrm{~cm}^{-1}$ [23,24]. Although most nozzle realizations use continuous (or near-continuous [25]) expansions [26,27], pulsed operation at low dutycycle into a buffer vacuum has shown a particularly high performance, because complete interferometric scans can be synchronized to intense macropulses without breaking the vacuum, if the time between pulses is used to re-evacuate the buffer [24]. This principle has been demonstrated in more than 50 applications [24,28-58] but it suffers from a single major disadvantage - the very large consumption of carrier gas (typically $99-99.99 \%$ of the expanded mixture) and of the admixed compound(s) to be investigated. As the best carrier gases ( $\mathrm{He}$ and $\mathrm{Ne}$ ) and some chemicals are quite costly and because isotope substitution is a valuable assignment tool, this can be a major obstacle for many research applications.

Complexes of monomeric organic molecules with single water molecules are among the best-studied gas phase cluster compounds [59-68]. The size-specific assignment of such microhydrates in the hydrogen-bonded $\mathrm{OH}$ stretching range of a linear infrared spectrum is usually straightforward, because their intensity varies rather linearly with the water and the organic molecule concentration and always slower than larger complexes with stagnation pressure. Due to the availability of several isotopologues of water, a broad repertoire of dynamical variations becomes accessible. A slit jet spectrometer with low consumption would already be valuable for this specific microhydrate application, and even more so for larger complexes, other solvent complexes, other spectral ranges, isolated molecules, their homodimers and -oligomers, as well as rare-gas coated molecules and clusters. If it were even moderately heatable, the number of accessible compounds would multiply and the clustering control would increase [69].

Here, we present a new gas-recycling slit-jet FTIR spectrometer which combines highest sensitivity in the mid- to near-IR spectral range with lowest available gas consumption. It allows for what we abbreviate as gratin-jet spectroscopy, where gratin stands for "gas-recycling atom-economic infrared" and also encodes an important additional feature, namely the heatability of the nozzle and vacuum chambers to minimize adsorbates (and thus a history of previously measured compounds) and to ultimately also study less volatile compounds. The spectrometer is based on a $700 \mathrm{~mm}$ slit nozzle which extends our earlier design of an adjustable $600 \mathrm{~mm}$ slit source with pre-expansion chamber [24], instead of the historical rotating-drum design [20] which was realized up to $500 \mathrm{~mm}$ slit length [70]. After describing the key ingredients of this new setup, we focus on its first applications to hydrate clusters [71], because traces of water anyway tend to accumulate after several hours of operation and thus provide a convenient natural concentration series for this cluster component. While this allows for maximum recycling yield, we also provide perspectives on future applications to water-free systems with more limited recycling of the gas flow. In those applications, the trace water accumulation still provides an automatic disambiguation from ubiquitous hydrate signals in cluster spectroscopy. This is achieved by comparing later phases of the measurement with earlier phases, but earlier renewal of the gas mixture is typically required for smallest water traces. It has to be stressed that only part of the capabilities of the new setup will be presented in this first account, awaiting further implemented options to be tested, developed and applied. 
We use the opportunity to contribute an improved direct-absorption perspective on a debate about the frequency of the hydrogen-bonded $\mathrm{OH}$ stretching fundamental of water dimer, which flared up recently after having been thought to be settled for a quarter of a century [72,73], correcting a wrong initial action spectroscopy assignment [74]. The consensus assignment from 1996 [72] was also confirmed by direct absorption spectroscopy [75,76], even using FTIR detection [77-79]. In contrast to its overtone [80-82], the hydrogen-bonded $\mathrm{OH}$ stretching fundamental of water dimer has an excellent IR visibility. Therefore, any action spectroscopy result should always be critically compared to direct absorption evidence. We provide purely experimental evidence that the consensus assignment from 1996 is still valid, and that some of the recent claims for the water dimer and trimer absorption spectra cannot be substantiated. For other systems such as the monohydrate of 1-phenylethanol, perfect agreement with previous action spectroscopy [63] is demonstrated.

\section{Materials and Methods}

\subsection{The Existing Jet FTIR Spectroscopy Setup}

The existing jet FTIR spectroscopy setup with a fine but lengthy slit nozzle (filet jet) forms the point of origin for the design of the new gratin (vide supra) jet setup. Therefore, a short description of its most important features is given in this section. A more detailed description can be found in Ref. [24].

To investigate cold and isolated small organic molecules and weakly bound molecular clusters, the filet jet setup is equipped with a $(600 \times 0.2) \mathrm{mm}^{2}$ long slit nozzle (Figure 1). Through this slit nozzle, mixtures of 1-2 analytes in low concentrations (ca. $0.01-1 \%$ ) in a carrier gas are expanded from a $67 \mathrm{~L}$ reservoir of higher pressure (typically 300-1200 hPa) into a vacuum chamber $\left(12-23 \mathrm{~m}^{3}\right)$. The resulting supersonic expansion is probed by FTIR spectroscopy. The vacuum chamber consists of a so-called "jet chamber", to which the nozzle is attached, and several vacuum buffers connected by pipes. Due to pressure build-up in the vacuum chamber during the gas flow, short pulses of 100-200 ms are combined with waiting times of 30-90 s for the pumps (1-2 three-staged rotary lobe pump assemblies with pumping capacities of $\left.(2000+500) \mathrm{m}^{3} \cdot \mathrm{h}^{-1}\right)$ to reduce the pressure in the vacuum chamber from $\sim 1 \mathrm{hPa}$ after a gas pulse to less than $0.2 \mathrm{hPa}$ before the next pulse.

For the IR spectroscopic investigation of the molecules and clusters in the supersonic expansion, the scans of a BRUKER IFS 66V/S spectrometer are synchronized to the gas pulses. The IR beam emitted from and modulated by the spectrometer crosses the expansion along the slit nozzle at a distance of $10 \mathrm{~mm}$ (nozzle to beam center). The nozzle length of $600 \mathrm{~mm}$ leads to a corresponding absorption path, facilitating measurements of compounds in low concentrations or of transitions with low IR visibility, such as overtones [29]. To slightly focus the IR beam along the absorption path, two lenses with focal lengths of $500 \mathrm{~mm}$ and $250 \mathrm{~mm}$ are installed in this sequence in the lateral walls of the jet chamber. For measurements in different spectral regions, different light sources ( $\mathrm{SiC}$ rod, tungsten lamp), beam splitters (mylar, $\left.\mathrm{KBr}, \mathrm{CaF}_{2}\right)$, optical lenses / windows $\left(\mathrm{KBr}, \mathrm{CaF}_{2}\right)$, and detectors (bolometer, $\mathrm{HgCdTe} / \mathrm{InSb}$ sandwich, InGaAs) can be employed.

Despite its successful application over the last 15 years [24,28-58], the setup suffers from several drawbacks. The spectrometer uses gain ranging [83] to increase the signalto-noise ratio $(\mathrm{S} / \mathrm{N})$ outside of the center burst region in the interferogram. However, the application of gain ranging is limited to optical velocities $\leq 80 \mathrm{kHz}$, effectively constraining the usable scan speed to this range. Furthermore, it is not possible to use both detector elements of the $\mathrm{HgCdTe} / \mathrm{InSb}$ sandwich detector simultaneously for measurements. The setup is not heatable, preventing measurements at elevated nozzle temperatures or of less volatile compounds with insufficient vapor pressure at room temperature. Another issue arises from the gas flow design. Due to constructive limitations, the nozzle had to be attached vertically to the jet chamber (see Figure 1). This effectively limits the expansion in the jet chamber to about $400 \mathrm{~mm}$ to preserve accessibility of the nozzle and the adjacent components in the laboratory. The subsequent wall leads to a local pressure build-up during the gas pulse, which can only be diluted sideways. To soften this back pressure and 
to allow for long pulses, the large buffer volume of up to $23 \mathrm{~m}^{3}$ is necessary, drastically increasing the spatial requirements of the setup.

The most important drawback of the existing setup, though, is the substance consumption during measurements. For example, for measurements in the NIR/MIR region at a typical stagnation pressure of $750 \mathrm{hPa}$, per pulse approximately $0.3 \mathrm{~mol}$ of the carrier gas helium (which makes up $99-99.99 \%$ of the gas mixture) flow through the nozzle. To obtain a single sample spectrum, 100-1000 spectrometer scans (and thus gas pulses) are typically averaged, and for a single analyte combination, 5-20 different spectra at varying conditions are measured. Due to impurities such as air or lubricant entering the expanded gas mixture at the pumping stage, it cannot simply be recycled to the reservoir and re-used for further pulses. This leads to a large substance consumption (typically one or more $50 \mathrm{~L}$ bottles of compressed rare gas and in excess of $1 \mathrm{~mol}$ of analyte per system), limiting the selection of analyte substances and carrier gases to commercially available and moderately priced compounds, at least for routine measurements.

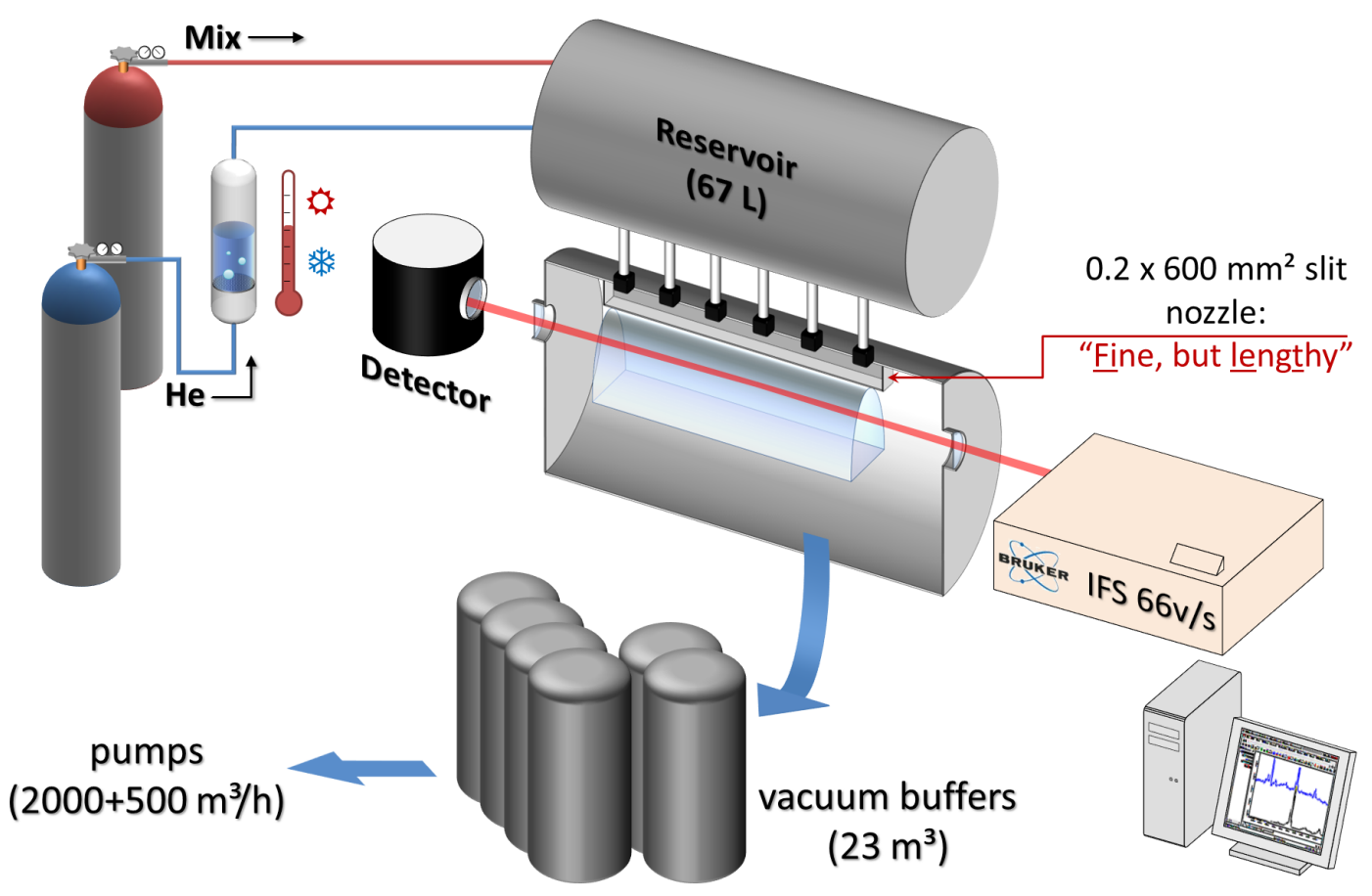

Figure 1. The filet jet spectroscopy setup. Taken from Ref. [84], licensed under CC BY-NC-ND 4.0.

\subsection{The New Gas-Recycling Jet FTIR Spectroscopy Setup}

The drawbacks of the existing filet jet setup were addressed at the design stage of the new jet FTIR spectroscopy setup. The main improvement is the implementation of full recycling of the once-expanded gas mixture, reducing substance consumption by up to two orders of magnitude (and even more in some favourable cases where the impurity buildup to be discussed later on is a feature) and thus allowing for the use of more expensive analytes and carrier gases, such as neon. This and the other main features of the gratin jet will be described in more detail in the following sections.

\subsubsection{The Gas-Recycling Concept}

The principle of the gas-recycling concept is simple: connect the "exhaust" of the pumps used for recompressing the gas mixture with the reservoir to recirculate the mixture. If the cycle of expansion, recompression and recirculation is repeated hundreds to thousands of times, a substantial reduction of substance consumption arises (vide infra). However, for the implementation of this concept, some issues need to be taken into account: 
- Ideally, no impurities such as purge gas, air, or lubricant aerosol should contaminate the gas mixture while being re-compressed by the pumps.

- Any trace impurities entrained in the gas mixture, e.g., via desorption, leakages, or at the pumps, accumulate during the gas-recycling operation. Requirements regarding leakage rate, evacuation procedure, but also stability of the chemicals are thus higher than in a conventional operation mode.

- The reservoir pressure directly corresponds to the pressure on the high-pressure side of the last pumping stage. To facilitate the use of different stagnation pressuresusually below atmospheric pressure-the pump in question needs to allow for an operation at such reduced pressures on the high-pressure side.

With these issues in mind, a setup consisting of a heatable reservoir, buffer volume, and gas recirculation together with a three-stage pumping station for gas-circulation and a turbomolecular pump for evacuation was purchased from PFEIFFER VACUUM GMBH. The setup offers a leakage rate of $\leq 1 \cdot 10^{-4} \mathrm{hPa} \cdot \mathrm{L} \cdot \mathrm{s}^{-1}$ while operating the pumps, allowing for continuous gas circulation over several hours before impurities accumulate in problematic quantities. The parts purchased from PFEIFFER VACUUM are combined with a $(700 \times 0.2) \mathrm{mm}^{2}$ slit nozzle designed and built in the workshop of the chemical department of Göttingen University and a BRUKER VERTEX 70v FTIR spectrometer to form the new gratin jet FTIR spectroscopy setup.

This setup is shown in Figure 2. For jet FTIR measurements, the gas mixture of $1-2$ analytes in a carrier gas is prepared in the reservoir $\left(0.2 \mathrm{~m}^{3}\right)$. At the start of the gascycle, a short pulse of the mixture is expanded through the slit nozzle into a compact buffer volume $\left(4 \mathrm{~m}^{3}\right)$ and probed by the mildly focused IR beam emitted from the spectrometer. The expanded gas mixture is recompressed by a series of two rotary lobe pumps and a screw pump and then recirculated to the reservoir. A turbomolecular pump can be used to evacuate the vacuum system between measurement campaigns, and this process can be supported by heating the main parts of the vacuum system up to $370 \mathrm{~K}$ via a heating system.

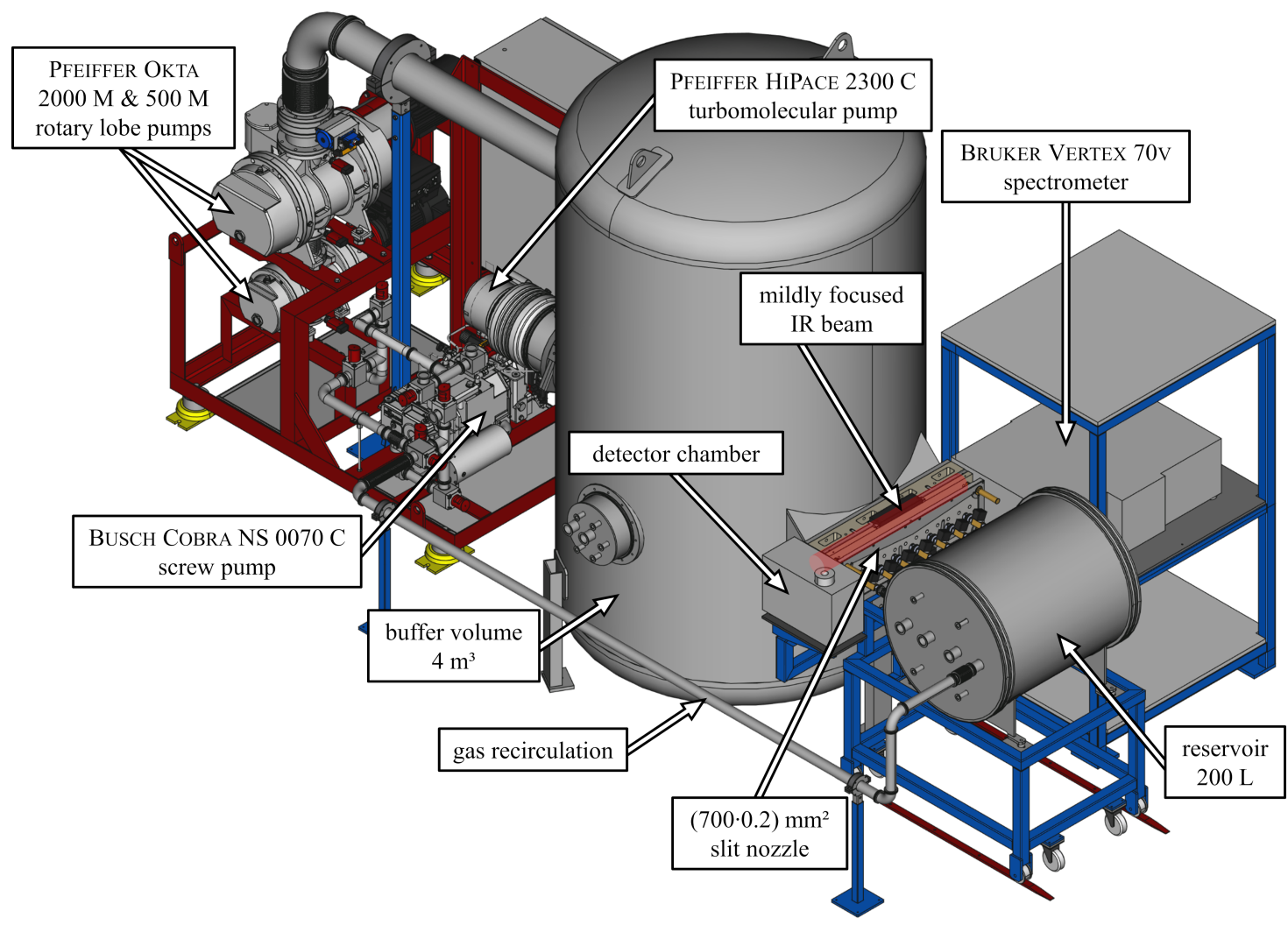

Figure 2. Overview of the gratin jet spectroscopy setup. Adapted from Ref. [85], licensed under CC BY 4.0. 


\subsubsection{Vacuum Technology}

To minimize desorption of unwanted substances into the gas mixture during gascirculation, the surface area of the vacuum system should be kept as low as possible. The first step towards this goal is to use the lowest possible volumes for reservoir and vacuum buffer. These volumes are determined by the throughput of the slit nozzle. To ensure sufficiently constant expansion conditions during the IR spectroscopic measurements, a maximum of $10 \%$ of the gas mixture in the reservoir should flow through the nozzle, per pulse. On the low pressure side, the background pressure at the end of the pulse should not exceed $2 \mathrm{hPa}$ to prevent the Mach shock from interfering with the area probed by the IR beam, at least for measurements of cold clusters and molecules, which are present in the zone of silence of the expansion. To achieve these goals, even for the longest pulses $(150 \mathrm{~ms})$ and highest stagnation pressures $(750 \mathrm{hPa})$, volumes of $0.2 \mathrm{~m}^{3}$ and $4 \mathrm{~m}^{3}$ for the reservoir and buffer volume, respectively, are sufficient. The drastic reduction of buffer size compared to the filet jet setup is possible due to an optimized gas flow design that reduces local pressure build-up at the nozzle (vide infra). Owing to practical limitations, cylindrical forms instead of surface-minimizing spherical vessels were chosen for reservoir and buffer (see Figure 2).

The vacuum scheme of the rotary lobe pump station used to recompress the expanded gas mixture is shown in Figure 3. The gas mixture, flowing from the vacuum buffer, is compressed to up to $10 \mathrm{hPa}$ at the first (PFEIFFER OKTA $2000 \mathrm{M}$ rotary lobe pump) and up to $50 \mathrm{hPa}$ at the second pumping stage (PFEIFFER OKTA $500 \mathrm{M}$ ). After the second stage, the gas mixture can either be further compressed by a third pumping stage (BUSCH COBRA NS 0070 C screw pump) or be directly recirculated to the reservoir while bypassing the screw pump. The pressures at the different stages can be estimated via three combined Pirani-capacitance PFEIFFER PCR 280 pressure gauges. The pump combination achieves a pumping speed of up to $2000 \mathrm{~m}^{3} \cdot \mathrm{h}^{-1}$ at inflow pressures of $1-2 \mathrm{hPa}$. Over longer periods of time, as they are necessary for the jet FTIR measurements, the second stage rotary lobe pump can be operated at outlet pressures of up to $30 \mathrm{hPa}$, and the screw pump at pressures between 300 and $1000 \mathrm{hPa}$. This allows for analogous stagnation pressures of up to 30 or $300-1000 \mathrm{hPa}$ for the supersonic expansion, corresponding to very mild or warm and increasingly colder expansion conditions, respectively.

To remove previous analytes, carrier gas, and impurities between measurement campaigns, the vacuum setup can be evacuated by a turbomolecular pumping station attached to the vacuum buffer, consisting of a PFEIFFER HIPACE $2300 \mathrm{C}$ turbomolecular pump (pumping speed: $1900 \mathrm{~L} \cdot \mathrm{s}^{-1}$ for $\mathrm{N}_{2}$ ) backed by a PFEIFFER ADIXEN ACP $40 \mathrm{G}$ multi-stage rotary lobe pump. This process can be supported by a mild bake-out (up to $370 \mathrm{~K}$ ) of the reservoir, nozzle, buffer and gas recirculation via a HORST GMBH heating system. The rotary lobe pump station is not heatable but reaches elevated temperatures during operation and can be closed off from the remaining vacuum system to avoid condensation during the bake-out process. While the rotary lobe pumps can be evacuated with the turbomolecular pump, such an evacuation is not advisable for the screw pump due to the risk of lubricant contamination. Therefore, the screw pump is closed off from the vacuum system during the evacuation process and is instead repeatedly purged with inert gases, such as nitrogen or helium, before and after each use.

Due to the gas-recycling concept, which recycles carrier gas and analytes as well as unwanted pollutants, care has to be taken to remove impurities from chemicals before the measurements. Custom-made stainless steel ports attached to the reservoir and vacuum buffer can be used to purify chemicals via repeated evacuate-flush or freeze-pump-thaw cycles. Carrier gas can be added to the gas mixture at the reservoir, buffer, or screw pump. 


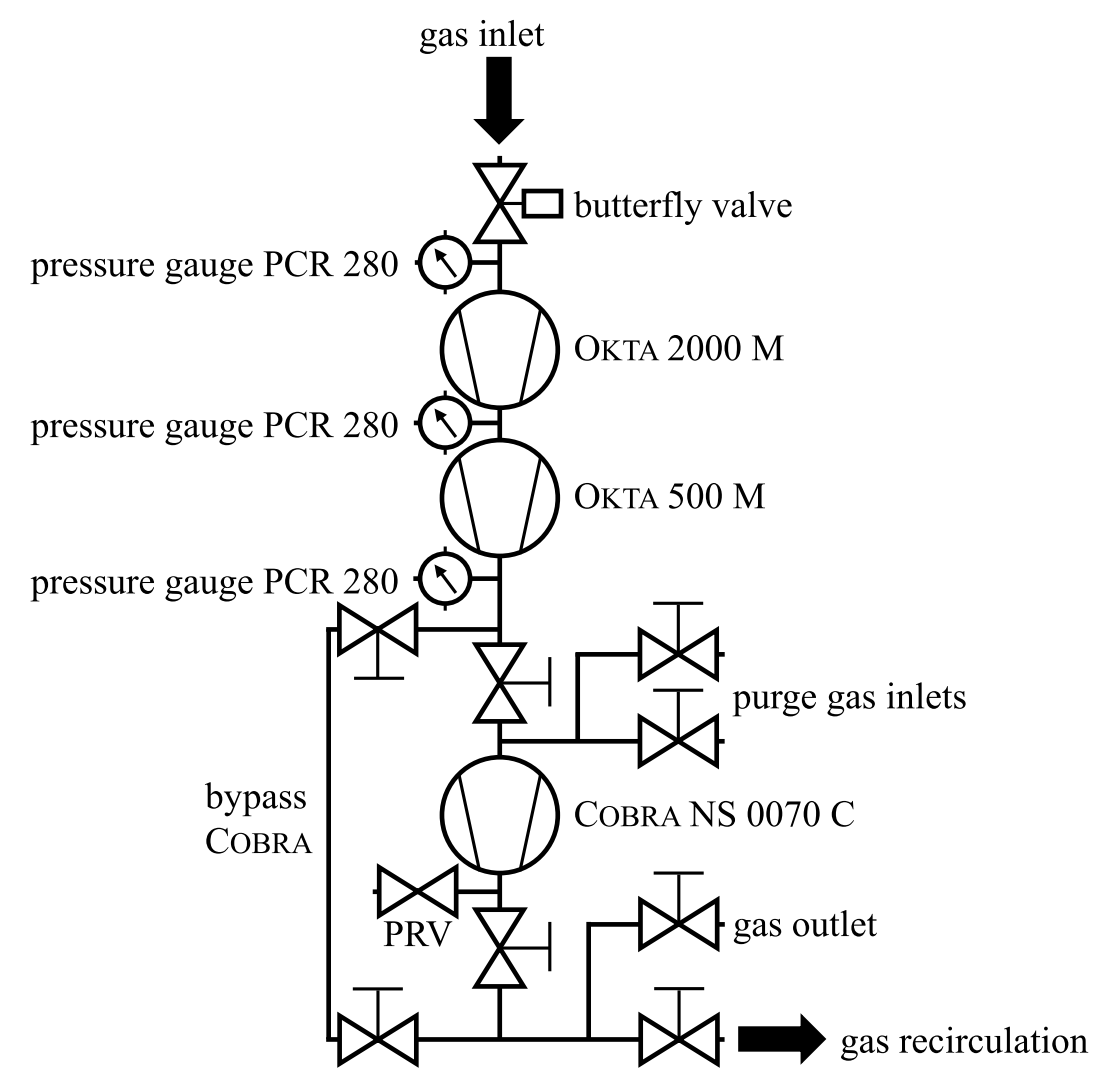

Figure 3. Vacuum scheme of the rotary lobe pump station. PRV: pressure relieve valve. Adapted from Ref. [86], licensed under CC BY-NC-ND 4.0.

\subsubsection{Slit Nozzle and Gas Flow Design}

The centerpiece of the new gratin jet setup is the $(700 \times 0.2) \mathrm{mm}^{2}$ slit nozzle. The design is based on the nozzle of the successful filet jet setup, scaled to the increased nozzle length of $700 \mathrm{~mm}$. The nozzle is connected to the reservoir via 7 feed lines, each containing a fast-switching solenoid valve (PARKER LUCIFER 221J3301E-299560-483816C2) to control the gas pulses. After passing the feed lines, the gas mixture flows into a pre-expansion chamber that ensures uniform gas flow along the whole nozzle length. The blades of the nozzle (see Figure $4 \mathrm{a}$ ) are angled by $35^{\circ}$ in the flow direction. All parts of the nozzle are made of stainless steel and it is electrically heatable.

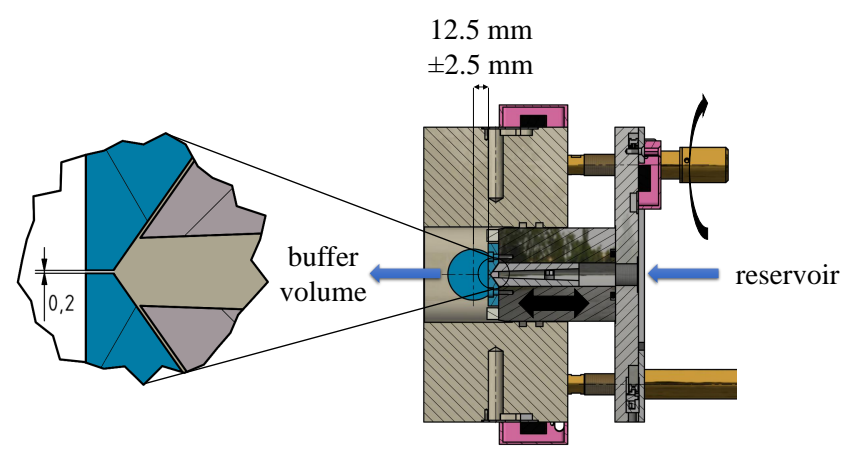

(a)

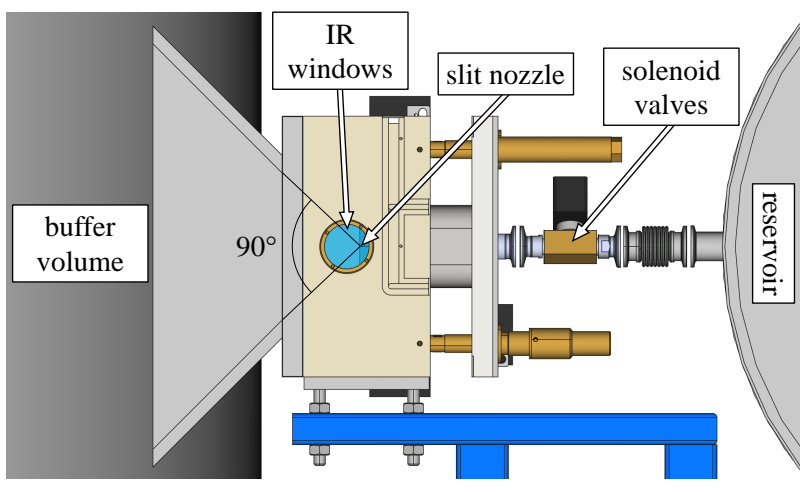

(b)

Figure 4. (a) Sideview of the slit nozzle. (b) Construction of nozzle and buffer volume, designed for optimized gas flow in the supersonic expansion. 
The nozzle corpus, consisting of the flanges for the connection with the feed lines, the pre-expansion chamber and the nozzle itself, is inserted into a second corpus containing the flanges for the IR windows/lenses and a rectangular flange for the connection to the buffer volume. Through the IR windows or lenses (blue circle in Figure 4a), the IR beam is coupled into the supersonic expansion along the length of the slit nozzle. The IR flanges have a diameter of $50 \mathrm{~mm}$ and are $776 \mathrm{~mm}$ apart, ensuring compatibility with the optical components used at the existing filet jet setup. However, due to the increased nozzle length at the gratin jet setup, now $90 \%$ of the IR absorption path between these flanges fall into the area of the supersonic expansion, compared to $77 \%$ at the previous setup.

To partially focus on different parts of the supersonic expansion, the distance between the nozzle and the center of the IR beam can be varied between 10 and $15 \mathrm{~mm}$ by moving the nozzle corpus relative to the IR corpus via a chain block mechanism (black arrows in Figure $4 \mathrm{a}$ ). The chain block mechanism enables a horizontal flow orientation from the reservoir through the nozzle into the vacuum buffer (blue arrows in Figure 4a) while still allowing to change the distance between nozzle and IR beam. Note that the adjustment range only covers a fraction of the mild IR focus area, but the sampling of regions close to the nozzle can still be varied. The horizontal integration of jet chamber and vacuum buffer enables a smaller buffer volume due to the absence of flow obstacles.

The section with the reservoir, nozzle, and buffer volume (right to left along the direction of the gas flow) is shown in Figure $4 \mathrm{~b}$ ). The base of the nozzle flange at the vacuum buffer is funnel-shaped, allowing for a free $90^{\circ}$ vertical angle for the expansion. Due to the attachment of the nozzle directly to the vacuum buffer, the distance between the nozzle and the opposing wall is $>1.5 \mathrm{~m}$. In combination, these optimizations avoid local pressure build-ups in the vicinity of the nozzle, thus allowing for the use of the compact buffer volume of only $4 \mathrm{~m}^{3}$, compared to the $12-23 \mathrm{~m}^{3}$ at the filet jet setup.

\subsubsection{IR Spectroscopy}

The evacuable BRUKER VERTEX 70V spectrometer used at the gratin jet setup is equipped with several light sources, beam splitters and detectors for measurements in different spectral regions and offers an optical resolution of up to $0.5 \mathrm{~cm}^{-1}$. Its detectorcentered digitization technology obviates gain-ranging for dynamic range enhancement and enables simultaneous two-channel detection for sandwich detectors. The available components together with the usable options for several parameters are summarized in Table 1.

As light sources, a Globar ( $\mathrm{SiC}$ rod) and two tungsten filaments can be used. Whereas the Globar and the less powerful $(50 \mathrm{~W})$ tungsten filament are installed internally (int.) inside the spectrometer, the more potent $(150 \mathrm{~W})$ tungsten filament sits inside a watercooled external (ext.) extension. The internal aperture and filter wheels are only usable with the internal light sources, whereas for the external tungsten filament, several circular apertures of varying diameters (see Table 1) and an additional filter mounting were custommade by our mechanical workshop. For jet measurements, an external detector port (inside the detector chamber, see Figure 2) is used. This can also be equipped with a Si-bolometer to extend measurements into the far infrared, assisted by the broadband beamsplitter and mirror optics, together with CsI windows and a cryogenic helium recovery port. Mechanical vibrations are not enhanced compared to the filet jet setup despite the more compact design. Therefore, an improved performance in this challenging and attractive spectral range [30] can be anticipated, but has not yet been explored.

Due to the limited maximum gas pulse duration (vide infra), high scan speeds and a low resolution are necessary to record a whole spectrum during a single pulse. Thus, detectors with fast response times are used for the jet FTIR measurements. For measurements in the NIR and MIR ranges (4000-600 $\mathrm{cm}^{-1}$ ), an INFRARED ASSOCIATES INC. detector with InSb and $\mathrm{HgCdTe}(\mathrm{MCT})$ detector elements in sandwich arrangement can be installed in the external detector chamber. A BRUKER InGaAs detector allows for measurements in the range above $4000 \mathrm{~cm}^{-1}$. Additionally, two BRUKER room-temperature DLaTGS 
detectors can be used to record broadband $\left(12,000-130 \mathrm{~cm}^{-1}\right)$ survey spectra, albeit at the price of lower detectivity and scan speeds. Thus, the latter are mostly used for reference measurements but not for jet spectra.

Since the waiting time between gas pulses decreases less than linearly with decreasing gas pulse duration, the best duty-cycle (measurement time per real time) is achieved by using the longest possible pulse duration. This duration is determined by the carrier gas, the volume of the buffer and the maximum tolerable background pressure $(2 \mathrm{hPa})$ during the gas pulse and corresponds to a value of 130-150 ms at the highest envisaged stagnation pressure of $750 \mathrm{hPa}$ for helium. To maximize efficiency, a high fraction of the gas pulse needs to be used for recording spectral data. With a pre-measurement time of $25 \mathrm{~ms}$ allowing for the gas pulse to fully form and a post-measurement time of $4 \mathrm{~ms}$ to avoid spectral artifacts due to electronic switching signals, 100-120 ms of the gas pulse can be used for IR spectroscopic measurements.

Table 2 lists the scan times $t_{\text {scan }}$ for recording IR spectroscopic data for several combinations of optical velocities and acquisition modes at a resolution of $2 \mathrm{~cm}^{-1}$. For the investigation of hydrogen-bonded clusters with their comparatively broad bands, such a moderate resolution is sufficient and avoids longer scan times and increased noise levels [24]. Forward-backward scan modes, in which the mirror changes direction during measurements, result in disproportionately long scan times and are thus less suitable for jet measurements. Therefore, all values listed in Table 2 correspond to forward-only scans. For the single sided modes, which record the interferogram to the desired resolution on just one side of the center burst, only an optical velocity of $80 \mathrm{kHz}$ offers a scan time in the optimum range (107 ms). For higher velocities, the available time for measurements is not efficiently utilized, whereas for lower velocities (not listed), the scan times exceed the upper limit given by the maximum pulse duration. For the double sided modes, which record the interferogram symmetrically to the full resolution on both sides of the center burst, the scan times are obviously higher than for the single sided modes. However, the second side of the interferogram needs to be measured to a lower resolution even in the single sided mode to allow for the phase correction [87]. As a result, in the double sided mode basically two interferograms can be recorded in less than twice the time of a single sided scan. For the optical velocities of 140 and $160 \mathrm{kHz}$, such a double sided scan is possible during a single gas pulse. At $140 \mathrm{kHz}$, the scan time falls in the optimum range $(105 \mathrm{~ms})$ for the compromise resolution of $2 \mathrm{~cm}^{-1}$. At lower optical velocities, the scan times are too long, whereas the scan time is below the optimum range for $160 \mathrm{kHz}$. In summary, double sided interferometer scans at a velocity of $140 \mathrm{kHz}$ constitute the optimum for the measurements of jet FTIR spectra at the gratin jet, but situations which require higher spectral resolution or involve other carrier gases may lead to variations.

Table 1. Currently available optical components and options [86].

\begin{tabular}{ll}
\hline Component/Parameter & Available Options/Values \\
\hline light source & Globar (int.); tungsten filaments: $50 \mathrm{~W}$ (int.), $150 \mathrm{~W}$ (ext.) \\
beam splitter & broadband, KBr, CaF $_{2}$ \\
IR windows $/$ lenses & $\mathrm{KBr}_{\mathrm{CaF}}$ \\
optical velocity $/ \mathrm{kHz}$ & $1.6,2.5,5,7.5,10,20,40,60,80,120,140,160$ \\
max. resolution $/ \mathrm{cm}^{-1}$ & 0.5 \\
int. aperture $/ \mathrm{mm}$ & $0.25,0.5,1,1.5,2,2.5,3,3.5,4,5,6,8$ \\
ext. aperture $/ \mathrm{mm}$ & $3,3.5,4,6,8,10,12,14,16,18$ \\
detector & LN-InSb/MCT-SW, RT-DLaTGS (2x), InGaAs \\
filter mountings & $\varnothing 25.4 \mathrm{~mm}$, filter wheel (int.) \& filter mounting (detector chamber) \\
\hline
\end{tabular}


Table 2. Scan times $t_{\text {scan }}$ of different combinations of optical velocity $v$ and acquisition mode at a resolution of $2 \mathrm{~cm}^{-1}$ [86]. Values in the optimum range (100-120 ms) are printed in bold.

\begin{tabular}{ccc}
\hline \multirow{v}{*}{$/ \mathbf{k H z}$} & \multicolumn{2}{c}{$t_{\text {scan }} / \mathbf{m s}$} \\
\cline { 2 - 3 } & Single Sided & Double Sided \\
\hline 80 & $\mathbf{1 0 7}$ & 184 \\
120 & 71 & 123 \\
140 & 60 & $\mathbf{1 0 5}$ \\
160 & 55 & 95 \\
\hline
\end{tabular}

\section{Results}

\subsection{Optics}

To optimize the signal-to-noise ratio $(\mathrm{S} / \mathrm{N})$ in different spectral regions, several combinations of light sources, apertures, and beam splitters were tested with the $\mathrm{InSb} / \mathrm{HgCdTe}$ sandwich detector and $\mathrm{CaF}_{2}$ windows at the spectrometer, jet chamber, and detector chamber. The spectral bandwidth of the light reaching the detector was limited to $4200-1200 \mathrm{~cm}^{-1}$ with an optical filter installed in the detector chamber. The performance of the different combinations was then determined by analyzing the noise levels in empty spectra. For this, the so-called Noise Test Challenge (NoTCh) routine is used: 1 minute of background spectra are measured through the empty jet chamber, followed by 1 minute of sample spectra under the same conditions. In the resulting absorbance spectra, the noise level is determined as the root-mean-square error $(R M S E)$ with respect to a quadratic fit of the spectral baseline in a moving $50 \mathrm{~cm}^{-1}$ window. The negative decadic logarithm of the $R M S E,-\lg (R M S E)$, is then plotted against the corresponding wavenumber.

The $-\lg (R M S E)$ plots for the two configurations with the best performance in the relevant spectral regions are shown in Figure 5. As expected, the combination of a $\mathrm{SiC}$ rod and a $\mathrm{KBr}$ beamsplitter (red) offers the lowest noise level (higher values for $-\lg (R M S E)$ ) for the $\mathrm{HgCdTe}$ detector element and the lower part of the spectral region probed with the $\mathrm{InSb}$ detector element. Uncompensated bands and lines of atmospheric carbon dioxide and water are counted as noise, thus reducing the $-\lg (R M S E)$ at their spectral positions (marked in Figure 5). For the $\mathrm{OH}$ and $\mathrm{CH}$ stretching regions, the combination of a $150 \mathrm{~W}$ tungsten filament and a $\mathrm{CaF}_{2}$ beam splitter leads to the lowest noise level (blue). The cross-over point between the two combinations shown in Figure 5 is located around $2700 \mathrm{~cm}^{-1}$. Thus, for measurements with a focus on the $\mathrm{OH}$ stretching region of the spectrum, as discussed below, the combination of the tungsten filament and $\mathrm{CaF}_{2}$ beam splitter is employed.

\subsection{Gas-Recycling}

The gratin jet setup is used in a gas-recycling mode, where the expanded gas mixture is recompressed and routed back to the reservoir after being expanded through the slit nozzle (vide supra). If a fraction $f_{R}$ of the gas in the reservoir is expanded in a single pulse, the recycling factor for $N_{\text {pulse }}$ gas pulses is $f_{R} N_{\text {pulse }}$. Typical values for $f_{R}$ are 0.05 for helium and 0.02 for neon, whereas $N_{\text {pulse }}$ can substantially exceed 1000, as will be shown below. Therefore, this mode leads to a significant decrease in substance consumption compared to single use operation. However, impurities entering the gas mixture via a leak or wall desorption can accumulate within the mixture over time.

When measuring in the gas-recycling mode over several hours, such an accumulation can indeed be observed (see Figure 6). In the $\mathrm{OH}$ stretching region of the jet spectra, three distinct lines rising in intensity with increasing gas-recycling duration can be observed (from light blue to dark blue). These lines can be assigned to ro-vibrational transitions (see Table 3) typical for jet-cooled water $(\mathrm{H})$. Their full width at half maximum is close to the nominal resolution despite the large aperture used. In unfavorable cases, such as the dilute ethanol spectrum shown in Figure 6, this leads to significant amounts of water in the gas mixture already after short periods of time, thus limiting the maximum usable duration 
of the recycling operation. Therefore, we tried to trace the dominant origin of the water accumulating in the gas mixture.

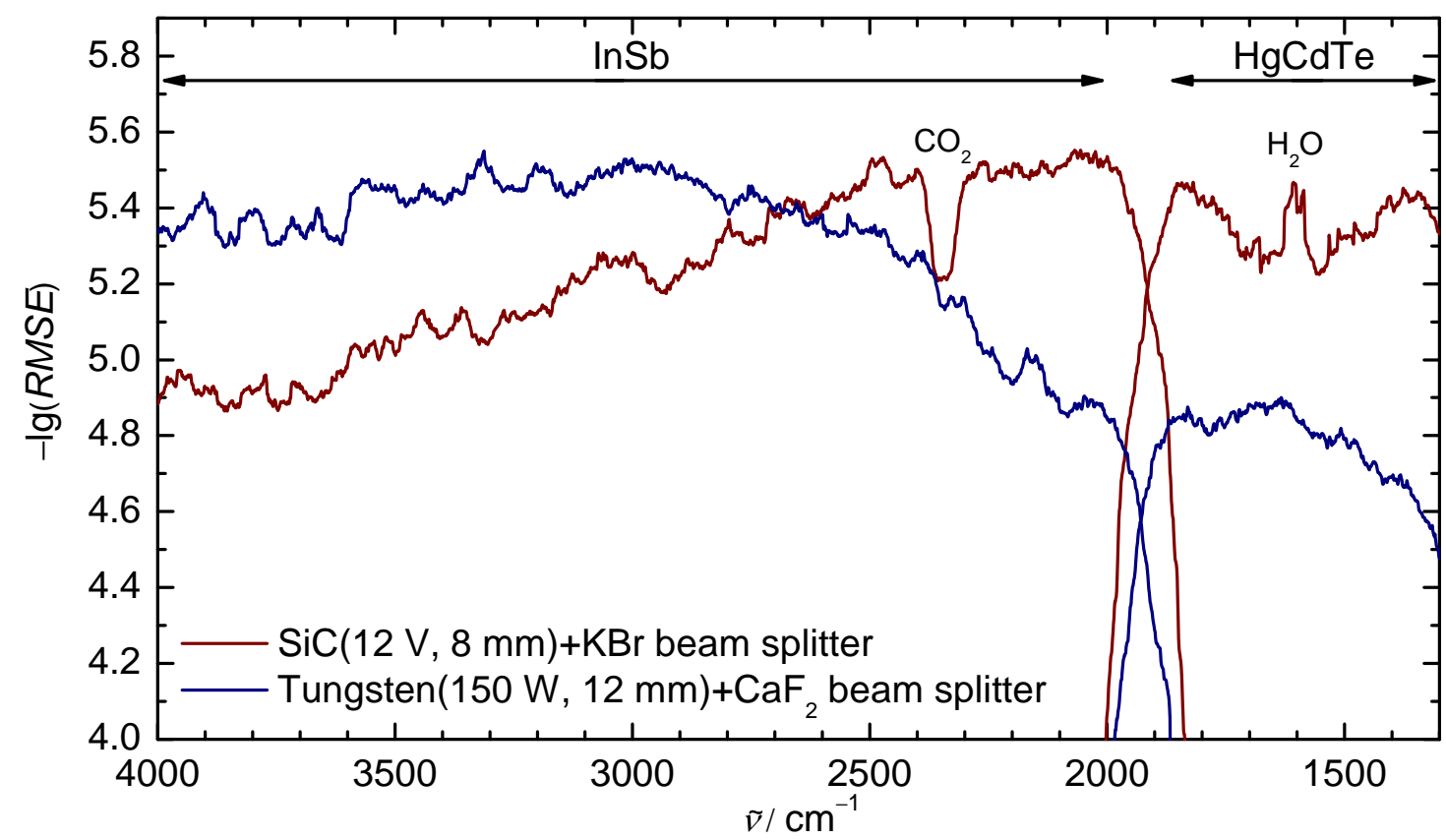

Figure 5. Sandwich detector performance with gratin optics, comparing the two channels for a tungsten source at $12 \mathrm{~mm}$ aperture and a $\mathrm{SiC}$ source at $8 \mathrm{~mm}$ aperture. Note that a higher value means lower noise and thus better performance.

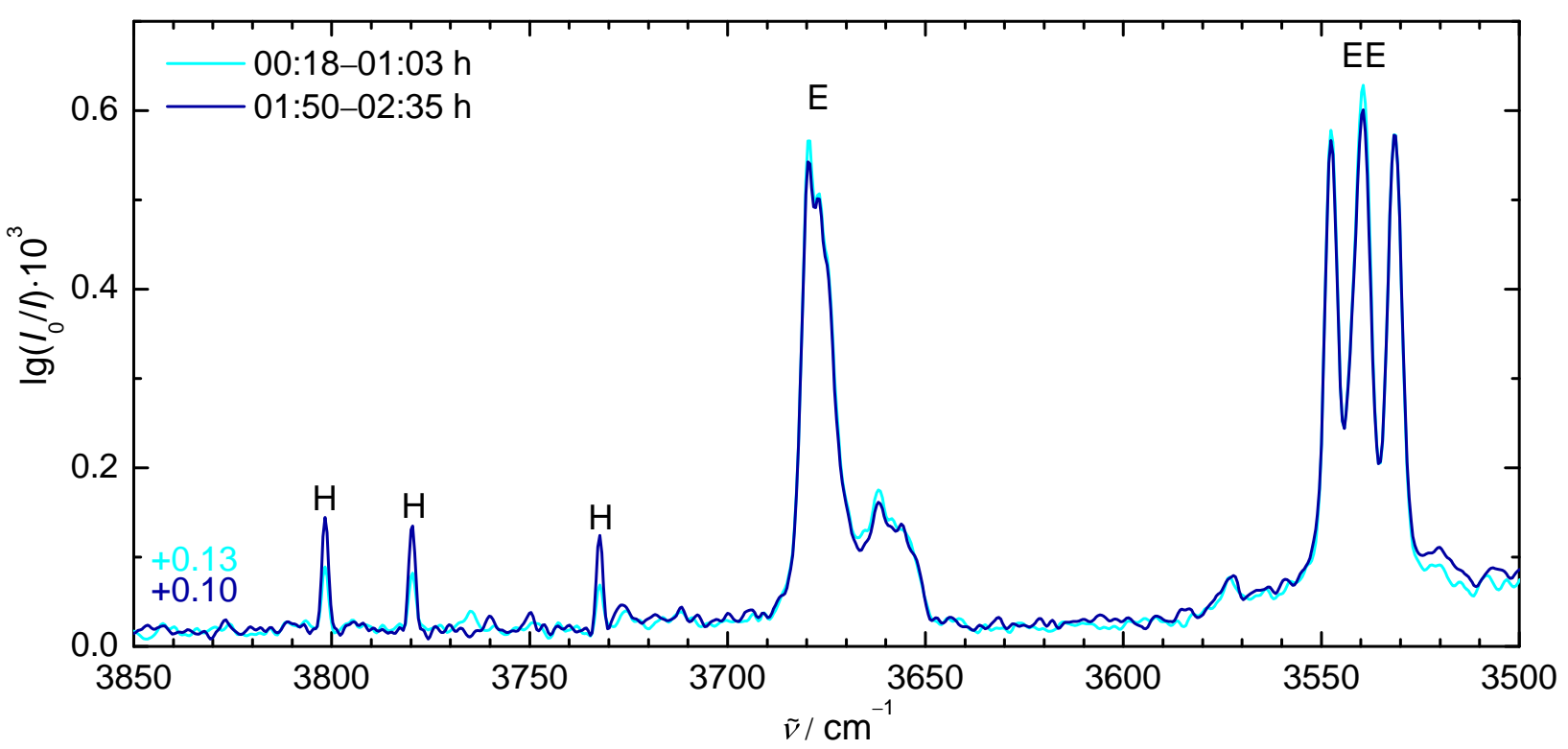

Figure 6. Rising intensity of rotationally cold water transitions $(\mathrm{H})$ during gas-recycling operation. With increasing time difference to the start of the recycling operation (light blue, to dark blue), the intensity of the monomeric water lines at 3802,3780 , and $3732 \mathrm{~cm}^{-1}$ increases in the spectrum of $1.30 \mathrm{hPa}(0.17 \%)$ ethanol (EtOH) in helium. The EtOH monomer (E) and dimer (EE) $\mathrm{OH}$ stretching bands are also marked in the spectrum. To illustrate the water increase, the baselines of the spectra were aligned near the water lines by displacing them along the ordinate, as indicated above the baselines. 
Table 3. Assignment of the observed ( $\left.\tilde{v}_{\text {exp }}\right)$ water lines [86] to literature values $\left(\tilde{v}_{\text {Lit }}\right)$ and their respective rovibrational transitions (Ref. [88]). In all cases, the vibrational transition involved is the asymmetric $\mathrm{OH}$ stretch fundamental. $J^{\prime}, K_{a}^{\prime}, K_{c}^{\prime}$, and $J^{\prime \prime}, K_{a}^{\prime \prime}, K_{c}^{\prime \prime}$ are the rotational quantum numbers of the excited and ground vibrational state, respectively. The $0.2(1) \mathrm{cm}^{-1}$ deviation from the literature line positions indicates a slight calibration error of the reference laser.

\begin{tabular}{cccccccc}
\hline$\tilde{\boldsymbol{v}}_{\text {exp }} / \mathbf{c m}^{-1}$ & $\tilde{\nu}_{\mathrm{Lit}} / \mathbf{c m}^{-1}$ & $\boldsymbol{J}^{\prime}$ & $\boldsymbol{K}_{a}^{\prime}$ & $\boldsymbol{K}_{c}^{\prime}$ & $\boldsymbol{J}^{\prime \prime}$ & $\boldsymbol{K}_{a}^{\prime \prime}$ & $\boldsymbol{K}_{c}^{\prime \prime}$ \\
\hline 3732.3 & 3732.13539 & 0 & 0 & 0 & 1 & 0 & 1 \\
3779.7 & 3779.49376 & 1 & 0 & 1 & 0 & 0 & 0 \\
3801.7 & 3801.41958 & 2 & 0 & 2 & 1 & 0 & 1 \\
\hline
\end{tabular}

One possible source is a leak of environmental air. Whereas the vibrations of oxygen and nitrogen are not visible in the IR spectrum and the fundamental vibrational transitions of carbon dioxide lie outside the spectral region initially probed at the gratin jet (4200-2450 $\left.\mathrm{cm}^{-1}\right)$, the humidity entrained in the air could lead to the lines observed in the $\mathrm{OH}$ stretching region of the jet spectrum. This is confirmed by deliberately adding $7.5 \mathrm{hPa}$ of environmental air to a mixture of $1.3 \mathrm{hPa}$ ethanol $(\mathrm{EtOH})$ and $741 \mathrm{hPa} \mathrm{He}$ (grey trace in Figure 7). At 3801,3780, and $3732 \mathrm{~cm}^{-1}$, jet water lines appear in the spectrum, similar to those observed after $\sim 2 \mathrm{~h}$ of recycling operation without air admixture (blue trace). However, at lower wavenumbers, the spectra differ significantly. The admixture of air leads to two major spectral effects in the $\mathrm{OH}$ stretching range that are not visible in the spectrum with the water-containing impurity: 1 . relaxation into the most stable ethanol dimer (homochiral all-gauche conformer) $[40,89,90]$ due to more efficient collisional cooling because of the heavier molecules in the air; 2 . bands at 3520 and $3508 \mathrm{~cm}^{-1}$, which can be assigned to $\mathrm{EtOH}+\mathrm{N}_{2}$ clusters [40]. A major air leak can thus be ruled out as the main source of the rising water amount in the circulating gas mixture.

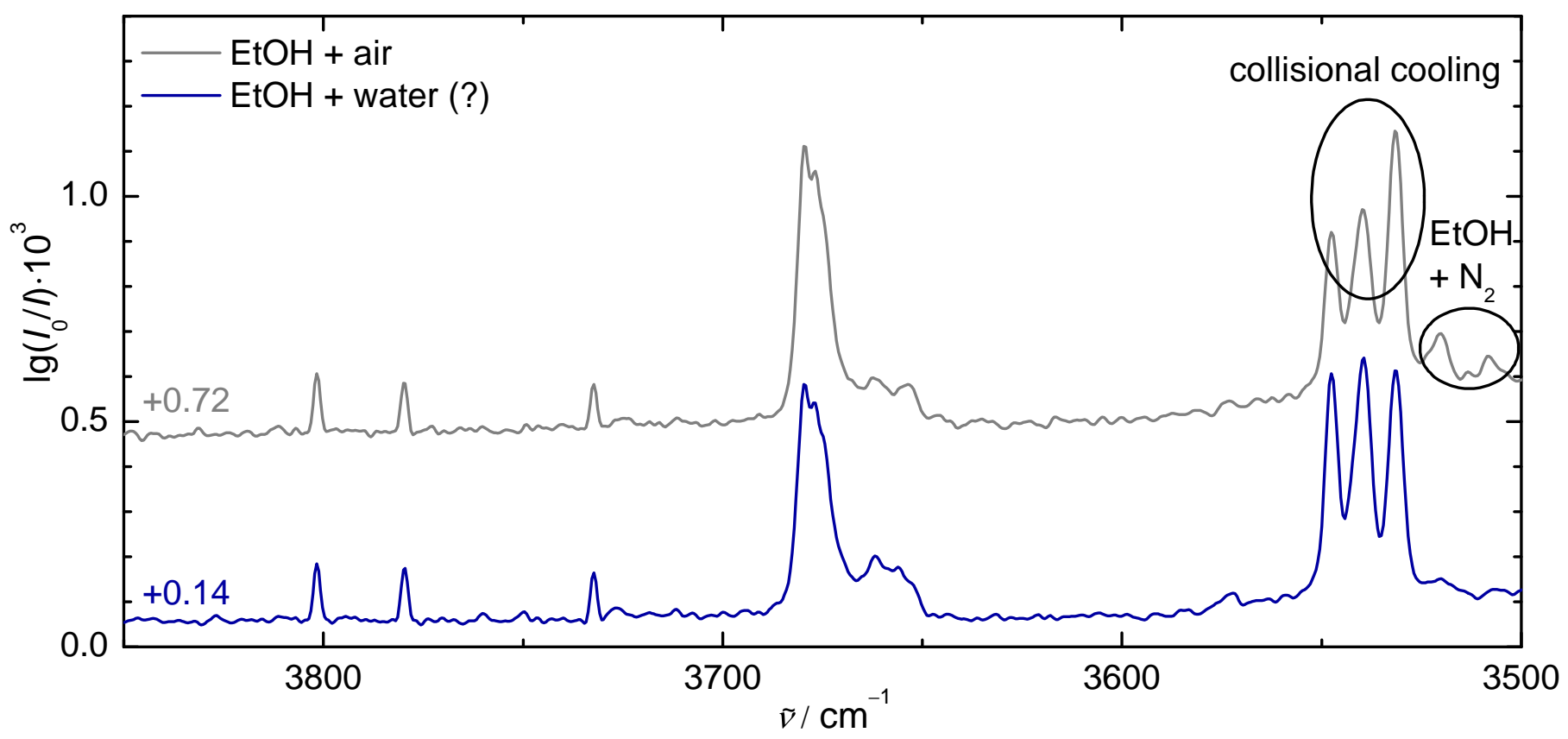

Figure 7. Ethanol gratin jet spectra with admixture of air (grey) or the rising water-containing impurity (blue). The spectra are displaced along the ordinate, as indicated above their respective baselines.

Another possible source for water would be a direct water leak from the internal cooling circuit of the screw pump. This pump uses a mixture of ethylene glycol and water as coolant. However, ethylene glycol was not detected in the jet spectra. Additionally, after partly deuterating the water in the coolant, neither $\mathrm{HDO}$ nor $\mathrm{D}_{2} \mathrm{O}$ were detected in the jet 
spectra, either. Since the screw pump is the only location with a direct contact between (liquid) water and the vacuum system, a direct water leak can also be ruled out as a source for the rising water amount observed in the jet spectra.

The most likely source of the water thus is desorption from the elastomeric seals and the walls of the vacuum chambers, tubes, and pumps. Several observations support this hypothesis:

- The water increase seems to be slower when the screw pump is bypassed. However, this is difficult to judge since only warm expansions with stagnation pressures of up to $30 \mathrm{hPa}$ are possible without the screw pump (vide supra). The screw pump is suspected to be one of the main sources for desorption, since it can only be flushed with inert gases and not be baked out under high vacuum conditions.

- $\quad$ Since the initial start of the operation of the gratin jet in December 2018, the growth rate of the water trace impurity has dropped considerably. Based on comparisons to jet spectra with a known amount of water (or to isolated bands of other compounds of known concentration within a spectrum), we estimate the increase rate in recent spectra to typically be $<0.5 \mathrm{mg} / \mathrm{h}$. First quantifications in March 2019 showed increase rates of $\sim 2 \mathrm{mg} / \mathrm{h}$ (cf. Figure 6). This reduction in trace water buildup could be explained by a lower water desorption rate due to the increasing time since the last full purging of the vacuum system.

- The new measurements show an initial release of 1-5 mg of water, depending on whether the preceding experiment involved high water concentrations. This initial release could be caused by the new analyte molecules replacing adsorbed water molecules at the walls and seals. After this initial release, the aforementioned water increase rate of $<0.5 \mathrm{mg} / \mathrm{h}$ sets in. Indeed, Figure 6 indicates a slight depletion of gaseous ethanol with water impurity buildup.

- After several hours, a saturation of the water concentration possibly sets in. However, this is difficult to judge due to the limited spectral resolution which does not allow for an exact quantification of two orders of magnitude more narrow rovibrational lines.

The increasing water amount effectively limits the duration for which the gas-recycling can be upheld before the reservoir needs to be refilled with new gas mixture. In favorable cases, the gas-recycling operation can be kept up over a whole workday with only tolerable amounts of water (and thus water clusters) accumulating in the gas mixture. This results in a significant decrease of substance consumption compared to the conventional single use operation at the filet jet.

Such a favorable case is the mixture of methanol $(\mathrm{MeOH})$ and 2-fluoroacetophenone (2FAP) in the carrier gas helium (He). Figure 8 shows the substance consumption $n$ dependent on the number of gas pulses $N_{\text {pulse, }}$ measured at the filet (green) and gratin (red) jet setups at a stagnation pressure of $750 \mathrm{hPa}$, respectively. Since He makes up more than $99.9 \%$ of the gas mixture, the He consumption $n_{\mathrm{He}}$ is used as a representation. For all calculations of the helium amount of substance, ideal gas behavior is assumed. At the filet jet setup, approximately $2.0 \mathrm{~mol} \mathrm{He}$ are needed to initially fill the reservoir $\left(V_{\mathrm{R}, \mathrm{fi}}=0.067 \mathrm{~m}^{3}\right)$ to the desired stagnation pressure. Subsequently, an additional $0.28 \mathrm{~mol}$ $\mathrm{He}$ is needed to refill the reservoir after each pulse, resulting in a linear increase of He consumption with increasing number of pulses (solid green line). At the filet jet, 150 gas pulses were measured, resulting in a total He consumption of $44.8 \mathrm{~mol}$. For comparison purposes, the He consumption is extrapolated (dashed green line) to the number of pulses (1500) measured at the gratin jet, which would result in a He consumption of 429 mol at the filet jet. 


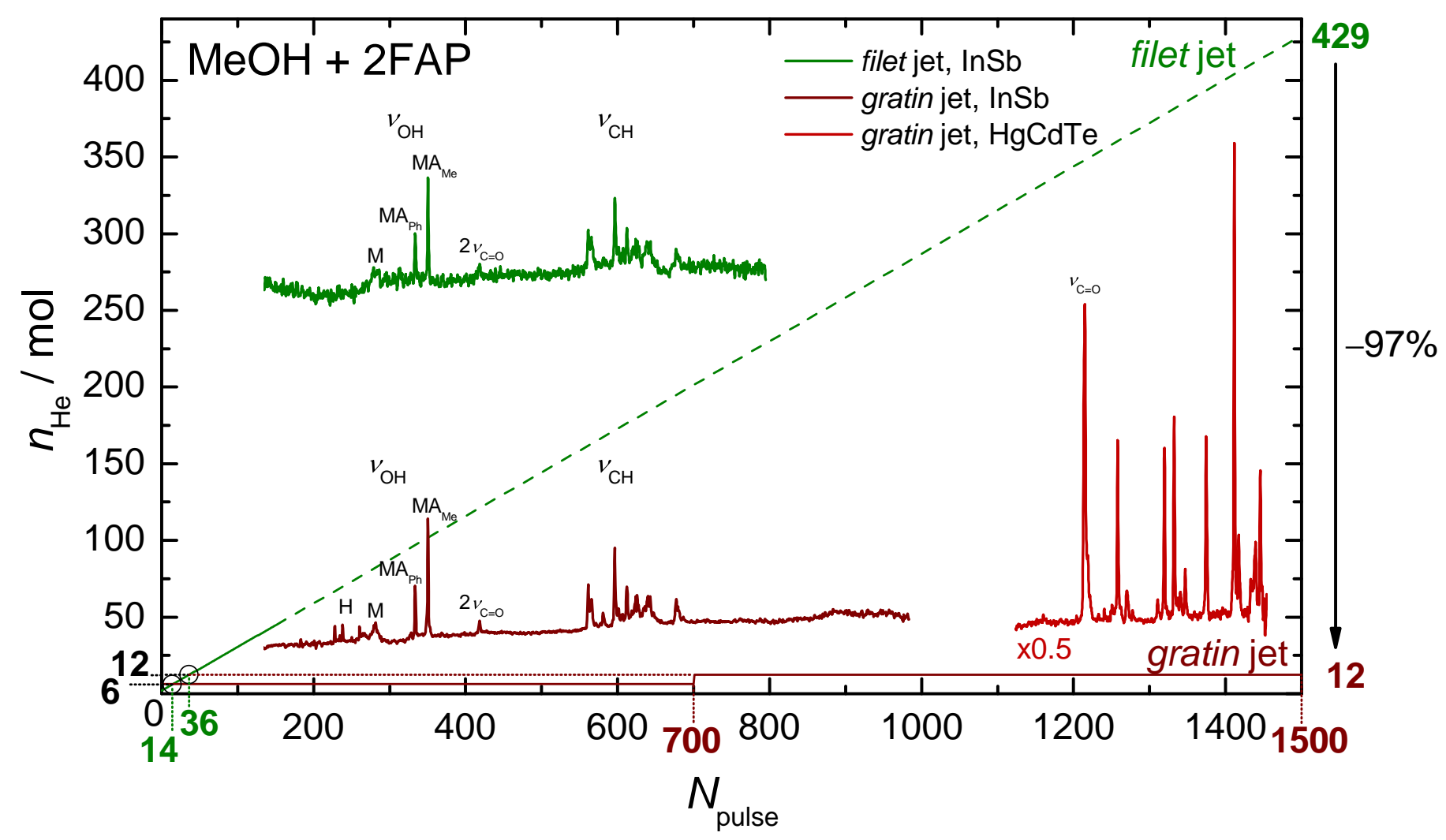

Figure 8. Comparison of helium consumption for measurements of $\mathrm{MeOH}+2 \mathrm{FAP}$ in $\mathrm{He}(>99.9 \%$ of the gas mixture) at the filet (green) and gratin (red) jet setups. At the filet jet, 150 pulses were measured (solid green line), and the helium consumption is extrapolated (dashed green line) to the 1500 scans measured at the gratin jet. At the gratin jet, the reservoir was filled twice, with the refill ocurring after 700 pulses (solid red line). The black circles indicate the intersections of the filet jet consumption line with the amounts of substance needed for one and two reservoir fillings at the gratin jet. The spectra recorded with a He consumption of $12 \mathrm{~mol}$ (36 pulses at the filet jet and 1500 pulses at the gratin jet) are shown as green (filet jet), dark red (gratin jet, InSb detector) and red (gratin jet, $\mathrm{HgCdTe}$ detector) traces. The spectral regions $\left(v_{\mathrm{OH}}: \mathrm{OH}_{\mathrm{H}}\right.$ stretching range; $v_{\mathrm{CH}}$ : $\mathrm{CH}$ stretching range) and most important absorption signals (H: water; $\mathrm{M}$ : methanol monomer; $\mathrm{MA} \mathrm{Me}_{\mathrm{Ph}}$ : methanol-2-fluoroacetophenone dimer, bound on the methyl $(\mathrm{Me})$ or phenyl $(\mathrm{Ph})$ side; $2 v_{\mathrm{C}=\mathrm{O}}: \mathrm{C}=\mathrm{O}$ stretch overtone; $v_{\mathrm{C}=\mathrm{O}}$ : $\mathrm{C}=\mathrm{O}$ stretch) are marked.

At the gratin jet, approximately $6.2 \mathrm{~mol} \mathrm{He}$ are needed to fill the reservoir $\left(V_{\mathrm{R}, \mathrm{gr}}=0.21 \mathrm{~m}^{3}\right)$. Due to the gas-recycling operation, after the initial filling no additional gas supply is required for measurements (red line), until the concentration of water in the gas mixture becomes problematic. In the example, the reservoir was evacuated and refilled after 700 pulses, and an additional 800 pulses were measured with this second filling. This results in a He consumption of $12 \mathrm{~mol}$ for measuring 1500 pulses. Compared to the $429 \mathrm{~mol}$ of He needed to carry out the same number of pulses at the filet jet, this corresponds to a decrease in substance consumption by $97 \%$. Conversely, with the amount of He needed to measure 1500 pulses at the gratin jet $(12 \mathrm{~mol})$, only 36 pulses ( $2 \%$ of 1500$)$ can be carried out at the filet jet. The spectra recorded with this He consumption are plotted in the figure. The most important signals in the $\mathrm{OH}\left(v_{\mathrm{OH}}\right)$ and $\mathrm{CH}\left(v_{\mathrm{CH}}\right)$ stretching ranges of the spectra measured with the InSb detector elements (filet: green, gratin: dark red) are marked. Additionally, the spectrum simultaneously measured with the $\mathrm{HgCdTe}$ detector element of the gratin jet setup is displayed in light red. A simultaneous $\mathrm{InSb} / \mathrm{HgCdTe}$ measurement is not possible at the filet jet (vide supra). As can be seen from the spectra, a significantly better $\mathrm{S} / \mathrm{N}$ per substance consumption is achievable at the gratin jet. However, when measuring several hundreds of pulses in the gas-recycling mode, water lines $(\mathrm{H})$ become visible in the gratin jet spectrum. An attempt to quantify the improved $\mathrm{S} / \mathrm{N}$, but also the influence of 
the water impurity on relevant $\mathrm{MeOH}+2 \mathrm{FAP}$ spectral features, is made in the following Section 3.3.

In summary, the source of the slowly increasing water amount in the circulating gas mixture has been carefully explored. A leak from the internal cooling circuit of the screw pump was ruled out by its partial deuteration. A dominant leak of environmental air was ruled out by the spectroscopic and spectrometric absence of other air components. We think that it is a sum of elastomeric seal and wall desorption, in particular from those parts of the screw pump which can only be flushed with dry gases, rather than baked out under high vacuum. Some saturation of the trace water influx appears to set in after many hours but is difficult to quantify due to the low resolution of the spectrometer. With this water influx being the limiting factor for the maximum duration of gas-recycling, the substance consumption for jet FTIR measurements can still be reduced by nearly two orders of magnitude compared to the conventional operation mode. This allows for the use of more expensive carrier gases, such as neon, and analyte substances, such as isotopologues or custom-made compounds.

\subsection{Fundamental Performance}

To examine the influence of the water impurity on the spectrum of the (binary) analyte combination of interest, one possibility is to look at the spectra of water with the individual analytes. Another possibility is to use water-free reference spectra of the analyte combination. For this purpose, the combination of $\mathrm{MeOH}$ and 2FAP can again serve as an example. Acetophenones, such as 2FAP, offer two attractive hydrogen bond docking sites for donors such as $\mathrm{MeOH}$ : the two lone pairs at the carbonyl oxygen atom. By substitution at the phenyl ring or the methyl group of the acetophenone, e.g., fluorination in the 2'-position of the phenyl ring (2FAP), the hydrogen binding preference between the lone pairs on the methyl $(\mathrm{Me})$ and phenyl $(\mathrm{Ph})$ sides can be influenced. This gives rise to a so-called intermolecular energy balance $[44,54,57]$, which can be used to benchmark quantum chemical energy differences by determining the abundance ratio of the Me- and Ph-bound mixed dimers in the supersonic expansion [44]. Therefore, the $\mathrm{MeOH}+2 \mathrm{FAP}$ donor $\mathrm{OH}$ stretch dimer bands $\mathrm{MA}_{\mathrm{Me}}$ (methyl side) and $\mathrm{MA}_{\mathrm{Ph}}$ (phenyl side) are of particular interest and we will focus on these bands to discuss the influence of the water impurity on the quality of the jet spectrum.

Panel (a) of Figure 9 shows the $\mathrm{OH}$ and $\mathrm{CH}$ stretching ranges of the spectrum of $\mathrm{MeOH}+2 \mathrm{FAP}$ recorded at the gratin jet (red) in comparison to a spectrum of 2FAP of similar concentration with water (blue), also recorded at the gratin jet. As can be seen, the dimer bands of $\mathrm{H}_{2} \mathrm{O}$ with $2 \mathrm{FAP}\left(\mathrm{HA}_{\mathrm{Me}}\right.$ and $\left.\mathrm{HA}_{\mathrm{Ph}}\right)$ partially overlap with those of $\mathrm{MeOH}$ with 2FAP $\left(\mathrm{MA}_{\mathrm{Me}}\right.$ and $\left.\mathrm{MA}_{\mathrm{Ph}}\right)$, thus subtly distorting the latter's intensities. This is due to the similar spectral $\mathrm{OH}$ stretching position of methanol and water complexes of ketones [31,91]. Note that the water concentration in the $\mathrm{H}_{2} \mathrm{O}+2 \mathrm{FAP}$ spectrum in a) is significantly higher than in the corresponding $\mathrm{MeOH}+2 \mathrm{FAP}$ spectrum, as evidenced by the intensities of the water lines marked " $\mathrm{H}$ ". As a consequence, the intensities of the HA dimer bands in the $\mathrm{H}_{2} \mathrm{O}+2 \mathrm{FAP}$ spectrum are also higher than in the MeOH+2FAP spectrum.

To quantify the degree of distortion of the $\mathrm{MA}_{\mathrm{Me}}$ and $\mathrm{MA}_{\mathrm{Ph}}$ band intensities by the water aggregates with $2 \mathrm{FAP}$, difference spectra can be formed. For this, subsets of the $\mathrm{H}_{2} \mathrm{O}+2 \mathrm{FAP}$ and $\mathrm{MeOH}+2 \mathrm{FAP}$ spectra with similar water concentrations are selected from the complete spectra (Figure 9b)). For the $\mathrm{H}_{2} \mathrm{O}+2 \mathrm{FAP}$ spectrum, the 200 scans with the lowest water concentration are used. Since the rate of the water increase was slower when recording the $\mathrm{MeOH}+2 \mathrm{FAP}$ spectrum, 700 scans of similar water concentration can be used for its subset. The difference spectrum (purple trace) results from subtracting the $\mathrm{H}_{2} \mathrm{O}+2 \mathrm{FAP}$ spectrum from the $\mathrm{MeOH}+2 \mathrm{FAP}$ spectrum. As evidenced by the absence of the water lines $(\mathrm{H})$ in the difference spectrum, the water compensation works well, whereas 2FAP is slightly overcompensated. Bands specific to 2FAP, such as the carbonyl stretch overtone $2 v_{\mathrm{C}=\mathrm{O}}$ and the $\mathrm{CH}$ stretch $v_{\mathrm{CH}}$, show small negative contributions to the difference spectrum. 




Figure 9. Influence of the water impurity on spectra of a binary mixture of MeOH and 2FAP in He. (a) spectrum (1600 scans) of 2FAP in He, with water impurity (blue); spectrum (1500 scans) of $\mathrm{MeOH}+2 \mathrm{FAP}$ in $\mathrm{He}$ (red). (b) subsets (see number of scans above the baselines) of the spectra in (a), containing similar amounts of the water impurity, and their difference spectrum (purple=red-blue). The most important spectral features are marked (H: water monomer; $\mathrm{M}: \mathrm{MeOH}$ monomer; $\mathrm{HA}_{\mathrm{Me} / \mathrm{Ph}}: \mathrm{H}_{2} \mathrm{O}+2 \mathrm{FAP}$ dimer, bound on methyl (Me) or phenyl (Ph) side; $\mathrm{MA} \mathrm{Me} / \mathrm{Ph}: \mathrm{MeOH}+2 \mathrm{FAP}$ dimer; $2 v_{\mathrm{C}=\mathrm{O}}: \mathrm{C}=\mathrm{O}$ stretch overtone; $v_{\mathrm{CH}}(\mathrm{A})$ : $\mathrm{CH}$ stretching bands of $2 \mathrm{FAP}(\mathrm{A})$ ). The spectra are displaced along the ordinate as indicated above their respective baselines.

By calculating the intensity ratio $I_{\mathrm{Me}} / I_{\mathrm{Ph}}$ of the mixed dimer bands, the influence of the water impurity can now be quantified. The ratio and its error are calculated as described in the supplementary material (IR) of Ref. [55]. However, instead of two researchers determining ratios based on integrations and peak heights independently, one researcher determines each ratio twice, using more narrow and wider bounds for the integrations, and peak heights with lower and upper bounds of the surrounding baseline as reference points.

Table 4 lists the resulting ratios for the full filet jet spectrum of MeOH+2FAP (150 coadded scans), the corresponding full gratin jet spectrum (1500 scans), and the difference spectrum described above. Compared to the esentially water-free reference spectrum recorded at the filet jet, the gratin jet spectrum displays a slightly lower intensity ratio, although the difference is not significant and lies within the error bars. The difference spectrum, for which the contributions of the water impurity were subtracted, shows an intensity ratio closer to the water-free reference, suggesting the reduction observed for the uncorrected gratin jet spectrum may have a real, non-statistical component and be caused by the water impurity. Note, however, that the noise introduced by the small number of scans in the difference spectrum largely washes out this small effect. Nevertheless, this highlights the fact that the influence of the increasing water amount on relevant spectral features of the analyte mixture has to be analyzed carefully, if the gas-recycling operation of the gratin jet setup is kept up over longer periods of time without refilling the reservoir. 
Table 4. Comparison of the performance of filet and gratin jet, using example spectra of MeOH+2FAP ( $N_{\text {scans }}$ : number of co-added scans per spectrum; $n_{\mathrm{He}}$ : amount of He used for recording the spectra; $\Delta t_{\text {real }}$ : real-time needed to record the spectra; $I_{\mathrm{Me}} / I_{\mathrm{Ph}}$ : intensity ratio of dimer donor $\mathrm{OH}$ stretch signals of Me and Ph sided dimer; filet jet spectrum: green trace in Figure 10a); gratin jet spectrum: red trace in Figure 8; gratin jet difference spectrum: purple trace in Figure 9).

\begin{tabular}{lllll}
\hline jet & $N_{\text {scans }}$ & $n_{\mathbf{H e}} / \mathbf{m o l}$ & $\Delta t_{\text {real }} / \mathbf{h h}: \mathbf{m m}$ & $\boldsymbol{I}_{\mathbf{M e}} / \mathbf{I}_{\mathbf{P h}}$ \\
\hline filet & 150 & 44.8 & $1: 18$ & $2.28 \pm 0.21$ \\
gratin & 1500 & 12.5 & $11: 30$ & $2.23 \pm 0.07$ \\
\hline gratin (diff) & - & - & - & $2.26 \pm 0.27$ \\
\hline
\end{tabular}

The upside of the gas-recycling operation is the greatly reduced substance consumption (vide supra). The data in Table 4 illustrates this: While using less than a third of the amount of substance, 10 times more scans can be recorded at the gratin jet, compared to the filet jet. This leads to a reduction of the error bars by a factor of 3 , again at less than $1 / 3$ of the substance consumption, albeit at the price of a greatly increased time demand for the measurement and the possible distortion of the intensity ratio by the accumulating water impurity.

Table 5 compares some relevant parameters of the FTIR measurements and the cycle of gas pulse and recovery period at the filet and the gratin jets. The time for one scan, i.e., the IR spectroscopic measurement of the sample (the gas mixture in the supersonic expansion), at the filet jet amounts to $100 \mathrm{~ms}$ with the typical settings for measurements in the $\mathrm{OH}$ stretching range $\left(80 \mathrm{kHz}\right.$, single sided, resolution $2 \mathrm{~cm}^{-1}$, InSb detector). At the gratin jet, with the optimized settings for measurements in this spectral range, each scan takes $108 \mathrm{~ms}\left(140 \mathrm{kHz}\right.$, double sided, resolution $2 \mathrm{~cm}^{-1}, \mathrm{HgCdTe} / \mathrm{InSb}$ detector in sandwich operation). Despite this longer scan time, the duration of the gas pulse, $t_{\text {pulse, }}$ could be shortened $(133 \mathrm{~ms})$ compared to the filet jet $(147 \mathrm{~ms})$. This is due to an optimization of the pre-measurement time, which allows for the supersonic expansion to fully develop before the sample measurement starts (vide supra). The shortened pulse also benefits the recovery period, i. e. the time needed for the pumps to reestablish a sufficiently low background pressure in the vacuum buffer before the next pulse. This applies despite the $17 \%$ longer slit nozzle in the gratin jet experiment. The total real-time for a complete cycle of helium gas pulse, measurements, and recovery time could be reduced by nearly $10 \%$, from $31 \mathrm{~s}$ at the filet jet to $28 \mathrm{~s}$ at the gratin jet. As a result, the duty cycle $\left(t_{\mathrm{scan}} / t_{\text {real }}\right)$ increases from $0.32 \%$ to $0.39 \%$.

Table 5. Comparison of relevant parameters of the FTIR measurements and the pulsed operation at the filet and gratin jets. $t_{\text {scan }}$ : Scan-time (time for data acquisition) of the IR spectroscopic (sample) measurement per pulse; $t_{\text {pulse }}$ : duration of the gas pulse; $t_{\text {real }}$ : real-time needed for a complete cycle of gas pulse, measurements, and waiting time between gas pulses; duty cycle: time used for measurements with respect to total time per cycle $\left(t_{\text {scan }} / t_{\text {real }}\right)$.

\begin{tabular}{lll}
\hline Parameter & Filet Jet & Gratin Jet \\
\hline$t_{\text {scan }} / \mathrm{ms}$ & 100 & 108 \\
$t_{\text {pulse }} / \mathrm{ms}$ & 147 & 133 \\
$t_{\text {real }} / \mathrm{s}$ & 31 & 28 \\
duty cycle $/ \%$ & 0.32 & 0.39 \\
\hline
\end{tabular}


To further compare the performance of the new gratin jet and the existing filet jet, the noise level in the example jet spectra of $\mathrm{MeOH}+2 \mathrm{FAP}$ in $\mathrm{He}$ can be analyzed. Since the two setups are not fully equivalent regarding the scan time per pulse, real-time per pulse, or substance consumption per pulse, a comparison of spectra with the same number of co-added spectrometer scans (and thus gas pulses) is not meaningful. Instead, we "scale" the gratin and filet jet spectra to the same value for one of those three quantities by taking a suitable subset of the recorded gas pulses.

The least advantageous comparison for the gratin jet is at the same total scan time, $\Sigma t_{\text {scan }}$, since the improvements regarding duty cycle, and-more importantly-substance consumption are ignored for this comparison. Due to the scan time per pulse being longer at the gratin jet, only the first 138 pulses of the gratin jet spectrum can be used to reach the same total scan time (15 s) as in the full filet jet spectrum (150 pulses). The upper panel of Figure 10a) displays the according spectra of $\mathrm{MeOH}+2 \mathrm{FAP}$ in $\mathrm{He}$, measured at the gratin (red) and filet jet (green). For the quantitative comparison of the noise level, the regions of the spectrum occupied by analyte bands can of course not be used (grey areas). However, signals of unwanted impurities such as water are counted as noise. As a measure of the noise level, we again use $-\lg (R M S E)$ (see Section 3.1). The lower panel of Figure 10a) shows this quantity for both spectra. Overall, the filet jet offers a slightly better noise level per scan time than the gratin jet, especially between 3500 and $3100 \mathrm{~cm}^{-1}$. This might be blamed on the sum of many subtle differences between the two jet spectrometers, such as the optical and scanner setup, the optical transmission, the beamsplitter properties, the electronic filtering and digitization, the scanning method, the detector and preamplifier response, electronic or mechanical perturbations, to name a few. It is partly offset by a larger absorption signal due to the longer slit nozzle and should not be considered very significant.

This changes drastically if all of the improvements of the gratin over the filet jet setup are taken into account by comparing at the same substance consumption, $\Sigma n$. Figure 10b) shows the comparison between gratin (red) and filet jet (green) for the lowest possible substance consumption at the gratin jet (one filling of the reservoir). With this amount of substance, 700 scans of individual gas pulses can be recorded at the gratin jet, compared to only 14 scans at the filet jet (vide supra). As expected, this leads to a significantly better noise level in the gratin jet spectrum, as is visible in the $-\lg (R M S E)$ values as well as in the spectrum itself. However, if the jet water lines are classified as unwanted noise, the noise level in the vicinity of these lines is aggravated almost to the filet jet level. This again highlights the fact that individual compromises between the highest possible savings regarding substance consumption (longest possible gas recycling duration) and the tolerable amount of water accumulation has to be found at the gratin jet. Further comparisons between gratin and filet jet performance can be found in the Supplementary Materials repository for this article [92]. 


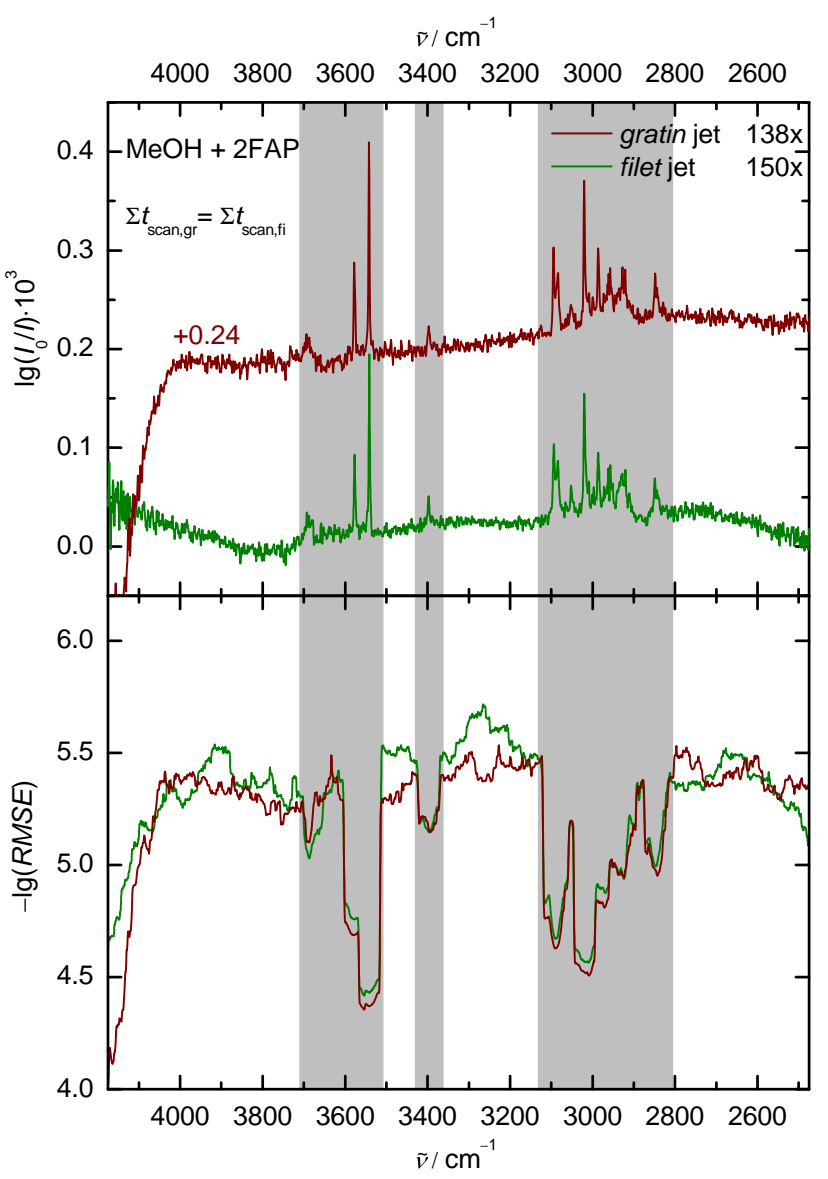

(a)

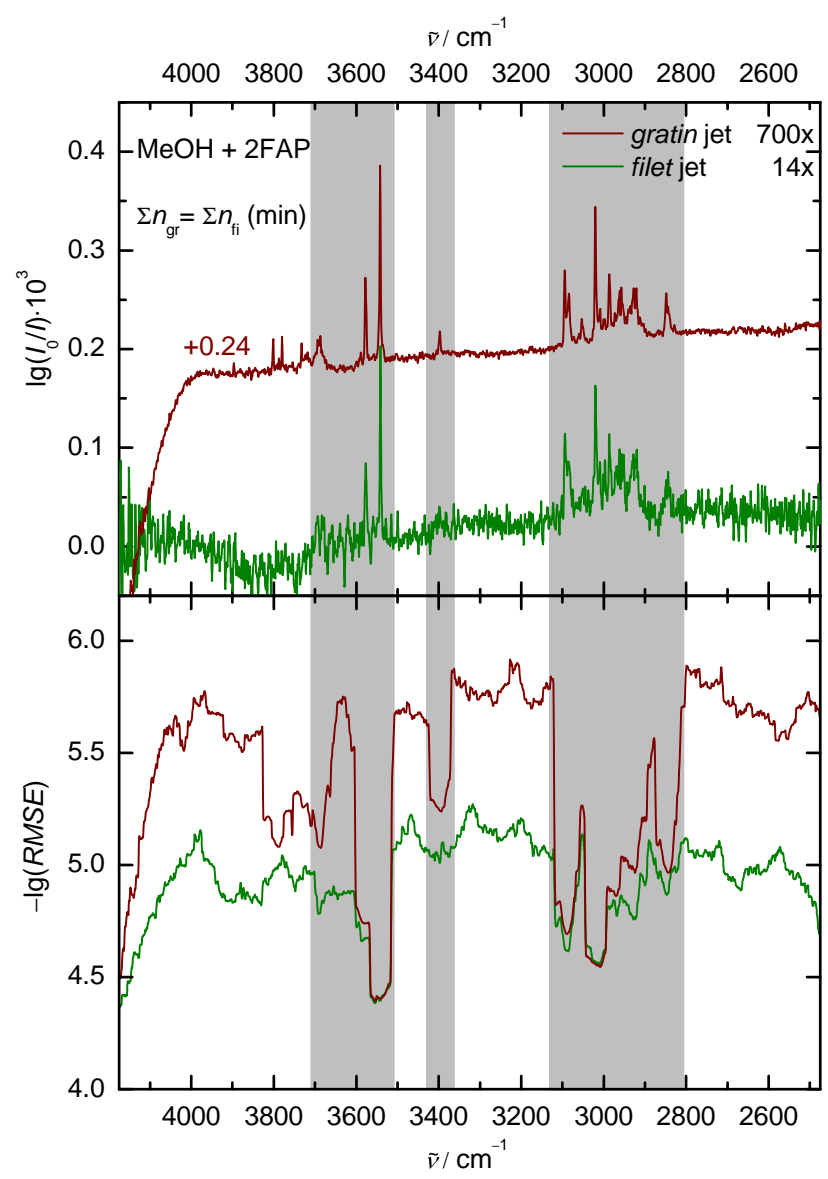

(b)

Figure 10. Upper panel, (a): comparison of spectra of MeOH+2FAP in He with the same total scan time $\Sigma t_{\text {scan }}$ (138 pulses at the gratin (gr, red, displaced by +0.24 along the ordinate), 150 pulses at the filet (fi, green) jet). Upper panel, (b): as (a), but for the same substance consumption $\Sigma n$ at filet and gratin jet. As a point of reference, the lowest possible consumption at the gratin jet ( 1 filling of the reservoir) was chosen. Lower panels: negative decadic logarithm of the root-mean-square error $(-\lg (R M S E))$ of the noise level in a $50 \mathrm{~cm}^{-1}$ moving window with respect to a quadratic fit of the baseline. The spectral regions occupied by absorption bands of the sample mixture, which cannot be used for the analysis of the noise level, are shaded in grey.

\subsection{Investigation of Hydrate Clusters}

The buildup of minute water contaminations in the recycled gas in particular in the test phase led to a first focus on hydrate clusters, because this buildup provides a natural size progression with time. The first signals emerging beyond those of the studied compound are due to monohydrates, followed by di- and oligohydrates after very long cycling times or explicit addition of extra water.

\subsubsection{Water Dimers vs. Trimers}

The conceptually simplest hydrates are those of the water molecule itself, i.e., water dimer and trimer. Due to a recent experimental challenge of the long-standing assignment of the hydrogen bonded $\mathrm{OH}$ stretching fundamental in the water dimer by a new form of action spectroscopy $[17,93]$, we felt it was timely to provide the currently best-possible FTIR direct absorption spectroscopy analysis in the most suitable carrier gas. We chose neon for the latter purpose, because it cools better than helium at low stagnation pressure, whereas for small water clusters there is still no risk of co-condensation on the clusters and due to the recycling concept, its use is affordable even for extended measurements. Note that in the following spectra, for simplicity we did not exploit the option of a higher duty cycle or resolution enabled by the higher pumping speed and smaller pulse intensity for 
the larger mass neon carrier gas. Instead, all pulse timings were chosen essentially identical to those optimized for helium.

Figure 11 shows the result of a straightforward and purely experimental water cluster size-discrimination strategy for both regular and heavy water (see the ESI of Ref. [91] for a preliminary analysis using fewer scans and partial pressures for regular water). Note that in the case of regular water, up to 1500 pulses were combined without refilling the containers, whereas in the case of $\mathrm{D}_{2} \mathrm{O}$, the gas mixture was renewed after about 300 pulses to reduce contamination by ordinary water desorbing from the walls. The initial growth of a given cluster size $n$ in the expansion with partial pressure $p_{w}$ of water differs in steepness between cluster sizes, all other things being equal. The steepness increases systematically with $n$ for sequential monomer addition. The growth law is not necessarily monomial $\left(N_{n} \sim p_{w}^{x_{n}}\right)$ due to the sequence of aggregation steps and if it is close to monomial, the exponent $x_{n}$ need not be equal to the cluster size $n$. Therefore, our primary analysis does not rely on such idealized behaviour. We compare three spectra at different partial water pressure $p_{w}$ between 0.4 and $1.1 \mathrm{hPa}$. One shows roughly equal signal strengths for postulated dimer and trimer peaks, another one is somewhat higher, the third one somewhat lower in partial pressure. This increases or decreases the trimer-to-dimer ratio with respect to the intermediate case. One way of analysing this different behaviour is to construct difference spectra in which either the dimer or the trimer signal is compensated. Such a sequence of three original and two difference spectra is shown in Figure 11. Spectrally distinct smaller and larger species can be discriminated based on the sign of their signal in the difference spectrum, if the partial pressure change is sufficiently large, but this is not the goal of the present study, which focuses on dimers and trimers. The spectral scaling relative to the optical density scale given in the upper right corner of each panel roughly matches the dimer intensity in the upper part and the trimer intensity in the lower part, with encircled compensation regions.

One can clearly see that the signal labeled 2 grows more slowly with $p_{w}$ than the signal labeled 3, such that signal 3 can be completely compensated in the upper difference spectrum and signal 2 in the lower spectrum. Only the sharp lines (sometimes clumped into broader structures due to the limited spectral resolution) survive more at high dilution (bottom spectrum) and these are well-known rovibrational transitions of the water monomer and its isotopologues. In the case of $\mathrm{D}_{2} \mathrm{O}$, there may also be a small upshifted HOD-donor dimer contribution $\left(2_{\mathrm{HOD}}\right.$ ?) due to small isotopic impurities accumulating during the recycling process, but it is too weak to be identified unambiguously. Evidently, the species contributing to signal 2 has less than about $10 \%$ signal contribution at band 3, and vice versa, except for the unlikely case that the shape of the overlapping bands is exactly the same for dimers and trimers and that the overlap is isotope-independent. The linear absorption spectrum of water dimer therefore does not contain significant intensity below about $3570 \mathrm{~cm}^{-1}\left(2610 \mathrm{~cm}^{-1}\right.$ for heavy water $)$ and the signal near $3533 \mathrm{~cm}^{-1}\left(2591 \mathrm{~cm}^{-1}\right.$ for OD) is essentially due to water trimers. The trimer satellite peak at $3548 \mathrm{~cm}^{-1}$ has been discussed in Ref. [91] and is missing (or shifted to the other side) for heavy water. To clearly discriminate trimer signals from downshifted larger clusters $(4,5)$, one would need spectra at higher partial pressures, but evidently there is a sizeable spectral gap due to the relaxed ring strain in these larger cyclic clusters and their enhanced cooperativity. These findings are in agreement with a large number of previous observations, some in linear absorption [75,77,79] or Raman scattering [94], others based on action spectroscopy [13,72,95,96]. Only some of the very early studies $[74,97,98]$ and, more surprisingly, the newest studies $[17,93]$ disagree with them. Our experimental data thus cannot support these latest reassignments of water dimer/trimer signals but instead strongly back the interpretation which prevailed over the last 25 years, based on the presented purely experimental linear absorption approach. 

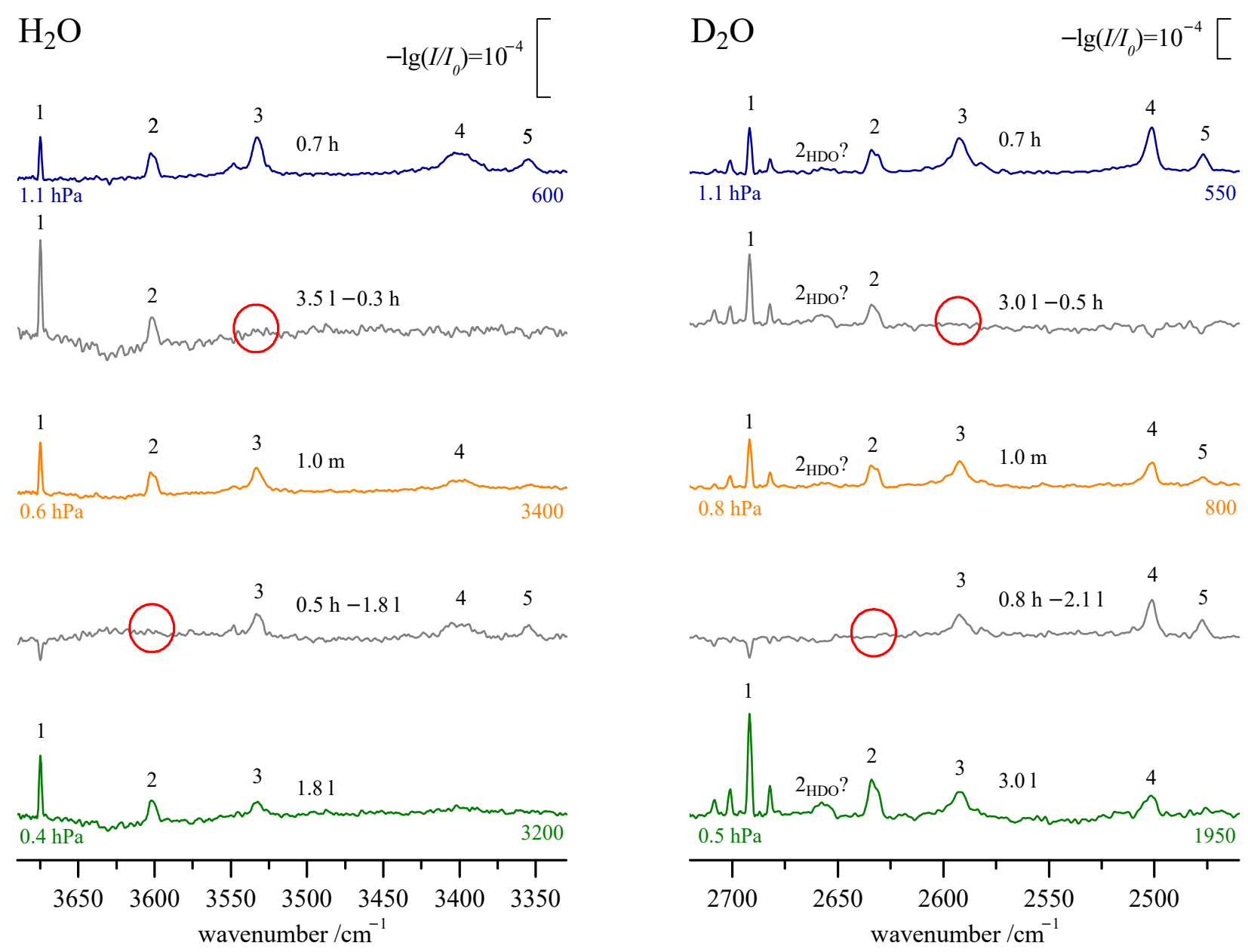

Figure 11. Separation of spectral contributions from $\mathrm{H}_{2} \mathrm{O}$ (left panel) and $\mathrm{D}_{2} \mathrm{O}$ (right panel) dimers (2) and trimers (3) in linear absorption supersonic jet spectra recorded in $750 \mathrm{hPa}$ neon expansions by comparing a high water partial pressure (h, top trace, $1.1 \mathrm{hPa}$ ) with a low partial pressure (l, bottom trace, 0.4 and $0.5 \mathrm{hPa}$ ) spectrum and taking their weighted differences such that the trimer (next-to-top) or dimer (next-to-bottom) contributions quantitatively vanish (circled areas). The spectra are intensity scaled to approximately match the dimer (upper part) or trimer (lower part) intensity of a medium partial pressure $(\mathrm{m}$, central trace, 0.6 or $0.8 \mathrm{hPa})$ spectrum. They also contain monomer transitions (1) and tetramer/pentamer contributions (4/5), the latter known to narrow down upon deuteration [75-78]. The number of co-averaged gas-pulses is given below the left end of each spectrum. Such spectra would be prohibitively expensive without recycling the neon gas (total gas flow of about 40000 standard liters).

Optionally, one may have a closer look at the actual scaling factors required to compensate a specific cluster size in Figure 11. For simple monomial scaling, they are consistent with a roughly 1.9-fold larger exponent $x_{n}$ for trimers than for dimers $\left(x_{3} / x_{2} \approx\right.$ $\log (3.5 / 0.3) / \log (1.8 / 0.5) \approx \log (3.0 / 0.5) / \log (2.1 / 0.8))$. Inserting the partial pressures $p_{w}$ (which carry a $0.1 \mathrm{hPa}$ uncertainty due to the co-addition of similar mixtures and slow concentration drifts), one furthermore obtains $x_{3}-x_{2} \approx 1.1$ for both isotopologues. This provides further evidence that the two signals differ by one water unit and are not due to the same species. Even the very unlikely case of perfect spectral overlap of dimers and trimers for regular and heavy water would contradict the findings of the recent reassignment, which actually claims different spectra for the two cluster sizes as well.

The somewhat more pronounced $[75,76]$ rotational sub-structure in the $\mathrm{D}_{2} \mathrm{O}$ dimer signal even allows for an estimate of its rotational temperature in the jet, despite the moderate resolution, by comparison to a higher-resolution spectrum [76]. With $\approx 10 \mathrm{~K}$, it is in line with expectations for a mild neon slit-jet expansion.

A final demonstration of the new capabilities for pure water clusters involves the cluster spectra of $\mathrm{H}_{2}{ }^{18} \mathrm{O}$, which have not been reported in vacuum isolation before. A 
single filling of $0.75 \mathrm{hPa}(120 \mathrm{mg})$ in $750 \mathrm{hPa}$ neon was used to record the spectra for 2700 gas pulses. For helium, this would correspond to a recycling factor of more than 100. For the more slowly flowing neon, the factor is currently only half as large, because the duty cycle was adopted from the helium experiments instead of being doubled. As shown in Figure 12, there is an increasing contamination by $\mathrm{H}_{2}{ }^{16} \mathrm{O}$, which can be removed reasonably well by subtracting $21-47 \%$ of the $0.6 \mathrm{hPa}$ expansion spectrum for ordinary water shown in Figure 11 (inverted bottom trace). The resulting subtraction spectra (based on the elimination of strong ordinary water monomer lines) are shown as central traces in Figure 12 for blocks of 900 pulses and their average is shown in the uppermost trace. Due to isotopic scrambling, the most reliable $\mathrm{H}_{2}{ }^{18} \mathrm{O}$ cluster band maxima are obtained and given for the first 900 pulses, where less than $20 \%$ contamination is found and subtracted, but the average over all 2700 pulses provides almost identical values. The isotopologue downshifts for the dimer and trimer signals amount to $\approx 10 \mathrm{~cm}^{-1}$, as one would expect for an $\mathrm{OH}$ stretching mode in $\mathrm{H}_{2}{ }^{18} \mathrm{O}$, and even the trimer satellite peak survives. The latter may provide further clues for its assignment.
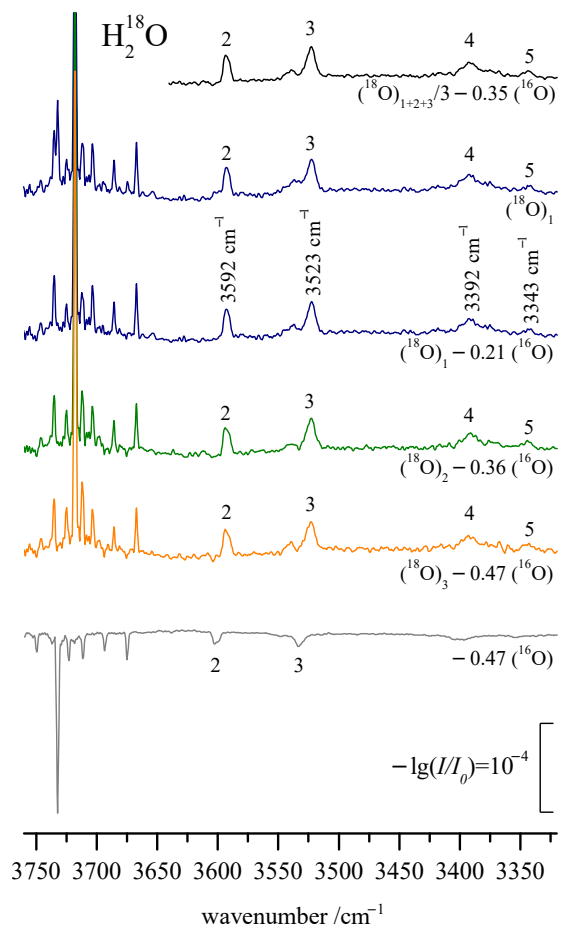

Figure 12. Clusters of $\mathrm{H}_{2}{ }^{18} \mathrm{O}(0.75 \mathrm{hPa})$ obtained in $750 \mathrm{hPa}$ neon expansions before (second trace from top) or after (next three traces) subtracting appropriate amounts from $\mathrm{H}_{2}{ }^{16} \mathrm{O}$ contributions (bottom trace, $0.6 \mathrm{hPa}$ ). The spectra from 2700 gas pulses are divided into three chronological blocks of 900 pulses ) 1,2,3 and co-averaged (top trace) after ordinary water subtraction. Cluster sizes 2,3,4,5 and band maxima in $\mathrm{cm}^{-1}$ are provided. Isotopic dilution during this 2-day single-filling experiment can be coped with by spectral subtraction.

In summary, the isotopic water cluster spectra show that one can cope with the contamination from desorbed ordinary water by either refilling the gas mixture after about 300 pulses (recycling advantage of about one order of magnitude compared to single use for the compound and the carrier gas) or by spectral subtraction for up to ten times more pulses (recycling advantage of about two orders of magnitude), depending on how much the $\mathrm{H}_{2}{ }^{16} \mathrm{O}$ interferes with the spectra. For $\mathrm{H}_{2}{ }^{16} \mathrm{O}$ clusters, the rare gas saving can in principle approach three orders of magnitude, compared to a series of spectra without gas recycling. 


\subsubsection{Ketone-Water Complexes}

The first published spectra obtained with the gratin jet setup refer to 1:1 ketone-water complexes, where a universal higher order resonance was uncovered [91]. The water complexes of 2FAP (vide supra) were part of this set and it is planned to extend the number of systems, also in view of a planned blind challenge on the predictability of water $\mathrm{OH}$ stretching vibrations in complexes with organic molecules. The gratin jet setup is ideal for this purpose. The organic compound is filled in under a high dilution in helium or neon and the first pulses reveal its $\mathrm{OH}$ spectral fingerprint and purity. Subsequent blocks of spectra show an evolution, where 1:1 hydrate complexes start to grow due to water desorption and eventually give way to 1:n complexes. The latter can be discriminated from the target monohydrates due to their different growth curves. If the impurity water buildup is too slow or saturates too early, extra water can always be added via the port of the buffer volume while keeping the gas mixture in the reservoir. In this way, hydrate cluster spectra under a wide variation of water concentration can be collected and the water concentration can always be monitored by the intensity of the three rovibrational antisymmetric stretching transitions surviving the jet cooling (see Table 3). After completion of the data collection for a single filling of single or double digit millimolar quantities of the organic target molecule, the system is evacuated and refilled at a different organic molecule concentration to optimize the signal and to rule out 2:1 cluster contributions.

\subsubsection{1-Phenylethanol-Water}

To further illustrate the capabilities and limitations of the recycling jet spectrometer, the 1:1 complex of 1-phenylethanol with water [63] shall be revisited. This complex is accessible to UV/IR double resonance spectroscopy, which can discriminate between different cluster sizes and also between conformations by probing (via holeburning) the IR spectrum which contributes to a certain UV transition. The 1:1-complex is thought to contain a hydrogen bond from the alcohol to the water and at least one of the $\mathrm{OH}$ groups of water binds back to the aromatic ring, forming a weak hydrogen bond. The other water $\mathrm{OH}$ is more free [63]. Therefore, the spectrum should consist of a strong, down-shifted band due to the alcoholic $\mathrm{OH}$ stretch and weaker, more weakly shifted water vibrational features which may be partially buried in the monomeric water lines. The advantage of UV/IR-spectroscopy is to remove any spectral contributions from the monomers and larger clusters and indeed, three signals attributed to the 1:1 complex were observed at $3565,3620,3733 \mathrm{~cm}^{-1}$, with similar signal strength [63]. The latter may be surprising, because hydrogen bonded $\mathrm{OH}$ groups are usually strongly enhanced relative to free or weakly bound $\mathrm{OH}$ groups in the IR spectrum, but there can be both instrumental and molecule-specific reasons for deviations from this rule.

Figure 13 shows gratin-jet spectra for $0.05 \mathrm{hPa}$ 1-phenylethanol $(0.007 \%)$ combined with $0.1,0.2$ and $0.4 \mathrm{hPa}$ water in $750 \mathrm{hPa}$ helium. Enantiopure 1-phenylethanol was used to reduce spectral complications from homodimers, which show up prominently above $0.01 \%$ admixture, but this is not relevant for the 1:1 complexes. The alcohol gives rise to a strong monomeric $\mathrm{OH}$ signal at $3648 \mathrm{~cm}^{-1}$, very close to the position in the UV/IR monomer selected signal [63] at $3647 \mathrm{~cm}^{-1}$ and the Raman signal [56] at $3648 \mathrm{~cm}^{-1}$. For the 1:1 complex, safe detection of the free $\mathrm{OH}$ stretch at $3733 \mathrm{~cm}^{-1}$ is impeded by overlap with a monomer transition at $3732 \mathrm{~cm}^{-1}$ (see Table 3), a clear disadvantage of the linear absorption technique. Subtraction experiments indicate a shoulder with substantial intensity near $3730 \mathrm{~cm}^{-1}$, which may be distorted by other hydrate clusters. In contrast, there are significant mixed cluster contributions at 3564 and $3619 \mathrm{~cm}^{-1}$, very close to the UV/IR signals of the 1:1 complex, together with some minor peaks increasing at higher water concentration. Therefore, our spectra confirm the band positions located two decades ago using a more sensitive fluorescence-dip technique, at similar $\mathrm{S} / \mathrm{N}$ ratio. We also confirm a comparable intensity of the two bands, with a roughly twice as strong signal at lower wavenumber. Within the available $\mathrm{S} / \mathrm{N}$ ratio, this intensity ratio is independent on concentration, thus supporting the same cluster origin of the two bands. Three signals emerging at higher 
water concentration are marked in the figure, because they closely correspond to the UV/IR assignments for the 1:2 complex [63]. For an assignment verification based on FTIR spectra, the concentration ratio would have to be extended beyond the 1:8 value shown in the most concentrated mixed spectrum.
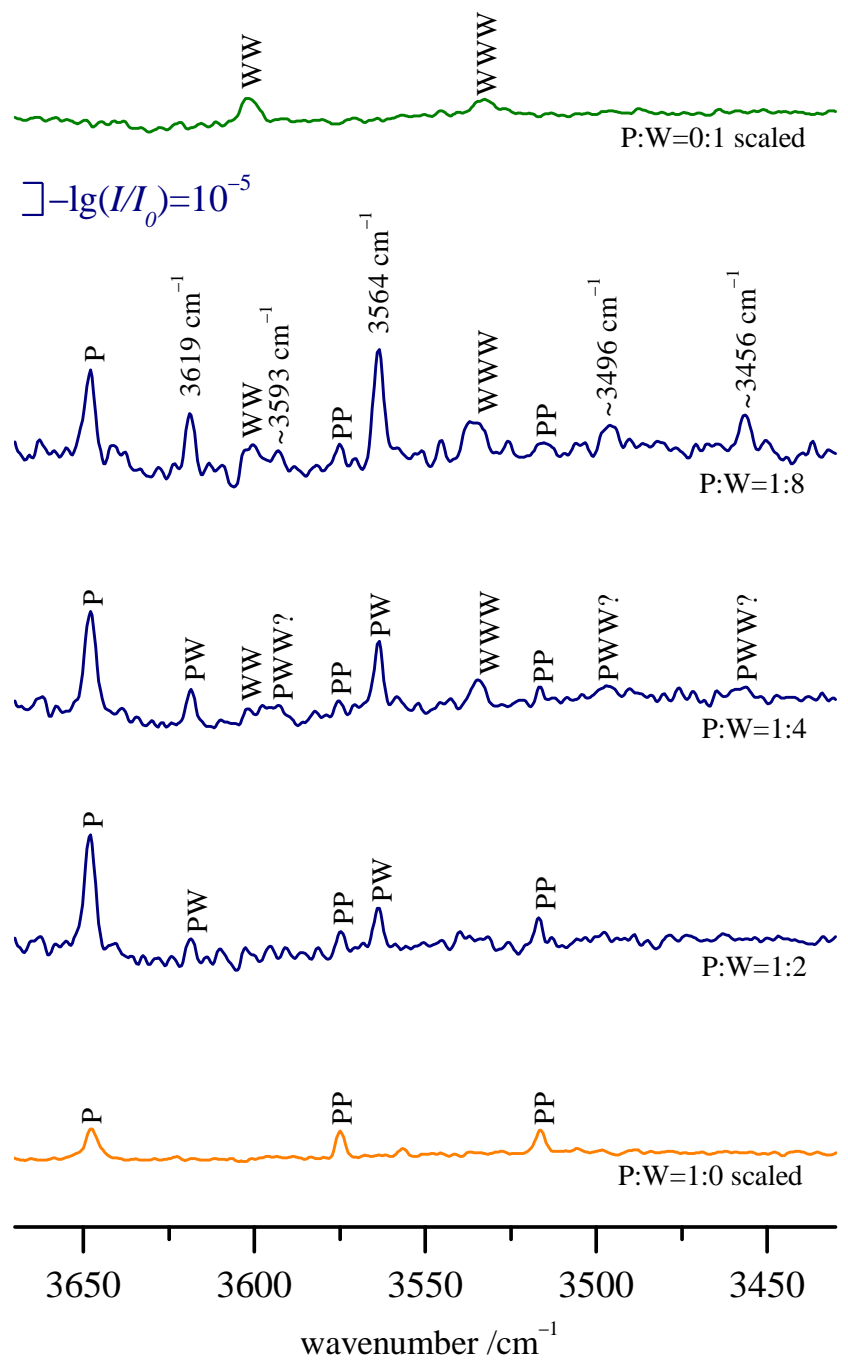

Figure 13. Confirmation of two hydrogen-bonded UV/IR fluorescence depletion $\mathrm{OH}$ stretching band centers [63] for the 1:1 hydrate PW of (enantiopure) 1-phenylethanol ( $\mathrm{P}, 0.007 \%$ in $750 \mathrm{hPa}$ helium), by increasing the water excess from twofold to eightfold (blue traces, 1000-2200 pulses each) with larger cluster signals showing up at higher concentration. Water dimers (WW) and trimers (WWW) as well as alcohol monomers and dimers (PP [56]) also contribute in this range, as shown by two qualitatively scaled spectral traces at the top and the bottom. PWW trimer labels are adopted from the fluorescence study [63] without further FTIR concentration scaling analysis.

This example shows nicely where the limits of our instrument are. Whenever there is spectral overlap or limited $\mathrm{S} / \mathrm{N}$, assignments may become uncertain and require further verification. On the other hand, the instrument is capable of confirming results from other techniques, such as the UV/IR band positions and relative intensities in the present case. Only a combination of several experiments is ultimately able to generate a high level of confidence about spectral assignments.

\section{Outlook}

Many options of the gratin jet spectrometer remain to be explored. Heavier carrier gases like $\mathrm{Ne}, \mathrm{Ar}$ or $\mathrm{N}_{2}$ not only allow for larger duty cycles and higher spectral res- 
olution (longer pulses) due to their higher viscosity and pumping efficiency, they also lead to larger clusters, to enhanced relaxation and to carrier-gas embedded clusters or nanomatrices $[40,49,77,79,89,99]$. Neon, in particular, offers a downscaling of the electrical power demand up to a factor of 2, by skipping the first compression stage without any significant compromise in the spectral quality or in the avoidance of carrier gas cocondensation. Nozzle heating [69] provides cluster size and isomerisation control [100]. Bypassing of the screw pump allows for very soft expansions which should provide a continuous transition from jet to equilibrium gas phase spectra and reveal details about thermal shifts [101,102]. If cold spots in the gas circulation are avoided, compounds with insufficient vapour pressure at room temperature for a jet expansion can be studied by electrical heating of the entire setup. Extension of the spectral range to the vibrational overtone [29] and fingerprint regions [24,30] will be feasible. Selective removal of water traces from the circulating gas by small-pore molecular sieves could be tried for clusters involving larger solvents. Among the most important perspectives is the possibility of systematic chemical substitution studies [28] using derivatives which are only commercially available in small amounts, because such chemical variations are among the most powerful tools to identify systematic trends and to rule out outliers. This is particularly valuable for weakly hydrogen-bonded complexes [32,33,38,51,52], where the relative effects can be large. More generally, it is important to vary the investigated molecular complexes in subtle ways before the spectroscopic assignments can be used for the benchmarking or even blind-challenging of theoretical methods $[42,45,53,55]$. A precautionary restriction of the present setup is that the investigated compounds should not corrode the gas-exposed surfaces, which are largely made of stainless steel. Even slight corrosion would likely increase the surface area and aggravate any desorption artifacts. Therefore, we currently refrain from studying strong organic acids or bases $[43,46,48,58]$ in the gratin jet FTIR cluster spectrometer and use the non-recycling filet variant [24] instead. Finally, the gas recycling concept could also be extended to other high-throughput jet spectrometers, in particular to Raman jet spectroscopy [94]. Multiexperimental approaches are absolutely essential for the securing of spectroscopic assignments $[36,37,39,41,47,50]$.

\section{Conclusions}

After 220 years of refinement [103], infrared absorption spectroscopy has now reached a status where small amounts of an arbitrary volatile compound can be used to study the vibrations of its cold 1:1 and larger hydrate or solvent complexes with high sensitivity and without interference from an embedding matrix. Even the softest matrices, such as helium nanodroplets, cause some spectral shifts $[100,104]$ and tend to stabilize metastable conformations [100,105], which complicates their use in the benchmarking of theoretical methods. By recycling the molecules and their carrier gas tens and hundreds of times through recompression of the gas mixture, expensive compounds such as isotope-enriched species also become accessible. Depending on the sensitivity to water impurities building up over time, an order of magnitude improvement of the signal-to-noise ratio is definitely possible, equivalent to two orders of magnitude in compound economy. Beyond being very useful on their own, the resulting linear absorption spectra can also be used to critically test action spectra of the same species, thus serving an additional experimental benchmarking purpose.

Supplementary Materials: The following are available online at Ref. [92], Figure S1: Comparison of filet and gratin jet spectra of $\mathrm{MeOH}+2 \mathrm{FAP}$ with the same total substance consumption, Figure S2: Comparison of filet and gratin jet spectra of $\mathrm{MeOH}+2 \mathrm{FAP}$ with the same total real time of measurements.

Author Contributions: Conceptualization, V.M., H.C.G., U.S. and M.A.S.; formal analysis, H.C.G.; investigation, H.C.G. and T.L.F.; resources, M.A.S.; data curation, H.C.G.; writing-original draft preparation, H.C.G. and M.A.S.; writing-review and editing, V.M., U.S., H.C.G., T.L.F. and M.A.S.; visualization, V.M., R.H., H.C.G., T.L.F.; supervision, M.A.S.; project administration, H.C.G. and M.A.S.; funding acquisition, M.A.S. All authors have read and agreed to the published version of the manuscript. 
Funding: The instrument used in this research was funded by the Deutsche Forschungsgemeinschaft (DFG, German Research Foundation) grant number 388444472, and the Niedersächsisches Ministerium für Wissenschaft und Kultur (Ministry for Science and Culture of Lower Saxony) grant number 76211-10-4/16 (05) (74ZN1583). The first benchmarking applications were funded by the DFG -389479699/GRK2455. The APC was funded by the Goettingen open access publication funds.

Institutional Review Board Statement: Not applicable.

Informed Consent Statement: Not applicable.

Data Availability Statement: Raw experimental data are either available via Ref. [92] or by email request.

Acknowledgments: We thank the mechanics (M. Zippert), electronics (A. Knorr) and glass workshops (A. von Roden) of the faculty for excellent support. This article is dedicated to the memory of Markus Gerhards, who has made pioneering contributions to the vibrational characterization of sizeand conformation-resolved organic hydrate complexes over wide spectral ranges and has always been a great inspiration and encouragement for our own work.

Conflicts of Interest: The authors declare no conflict of interest. The funders had no role in the design of the study; in the collection, analyses, or interpretation of data; in the writing of the manuscript, or in the decision to publish the results.

Sample Availability: All compounds were obtained commercially and samples of the compounds are not available from the authors.

Abbreviations
The following abbreviations are used in this manuscript:
$\begin{array}{ll}\text { DLaTGS } & \text { Deuterated L-alanine doped triglycine sulfate } \\ \text { 2FAP } & \text { 2'-Fluoroacetophenone } \\ \text { FTIR } & \text { Fourier-Transform Infrared } \\ \text { IR } & \text { Infrared } \\ \text { MCT } & \text { HgCdTe } \\ \text { MIR } & \text { Mid IR } \\ \text { NIR } & \text { Near IR } \\ \text { NoTCh } & \text { Noise Test Challenge } \\ \text { RMSE } & \text { Root-Mean-Square-Error } \\ \text { RT } & \text { Room temperature } \\ \text { S/N } & \text { Signal-to-Noise } \\ \text { UV/IR } & \text { Ultraviolet-Infrared (action spectroscopy) }\end{array}$

\section{References}

1. Nesbitt, D.J. High-Resolution, Direct Infrared Laser Absorption Spectroscopy in Slit Supersonic Jets: Intermolecular Forces and Unimolecular Vibrational Dynamics in Clusters. Annu. Rev. Phys. Chem. 1994, 45, 367-399. [CrossRef]

2. Buck, U. Structure and Dynamics of Small Size Selected Molecular Clusters. J. Phys. Chem. 1994, 98, 5190-5200. [CrossRef]

3. Castleman, A.W.; Bowen, K.H. Clusters: Structure, Energetics, and Dynamics of Intermediate States of Matter. J. Phys. Chem. 1996, 100, 12911-12944. [CrossRef]

4. Bačić, Z.; Miller, R.E. Molecular Clusters: Structure and Dynamics of Weakly Bound Systems. J. Phys. Chem. 1996, 100, 12945-12959. [CrossRef]

5. Zwier, T.S. The Spectroscopy of Solvation in Hydrogen-bonded Aromatic Clusters. Annu. Rev. Phys. Chem. 1996, 47, 205-241. [CrossRef]

6. Ebata, T.; Fujii, A.; Mikami, N. Vibrational spectroscopy of small-sized hydrogen-bonded clusters and their ions. Int. Rev. Phys. Chem. 1998, 17, 331-361. [CrossRef]

7. Buck, U.; Huisken, F. Infrared spectroscopy of size-selected water and methanol clusters. Chem. Rev. 2000, 100, 3863-3890. [CrossRef]

8. Buck, U.; Huisken, F. Additions and Corrections: Infrared spectroscopy of size-selected water and methanol clusters. Chem. Rev. 2001, 101, 205-206. [CrossRef] [PubMed]

9. Matsuda, Y.; Mikami, N.; Fujii, A. Vibrational spectroscopy of size-selected neutral and cationic clusters combined with vacuum-ultraviolet one-photon ionization detection. Phys. Chem. Chem. Phys. 2009, 11, 1279-1290. [CrossRef] 
10. Schwing, K.; Gerhards, M. Investigations on isolated peptides by combined IR/UV spectroscopy in a molecular beam - structure, aggregation, solvation and molecular recognition. Int. Rev. Phys. Chem. 2016, 35, 569-677. [CrossRef]

11. Lee, Y.F.; Kelterer, A.M.; Matisz, G.; Kunsági-Máté, S.; Chung, C.Y.; Lee, Y.P. Infrared absorption of methanol-water clusters $\left(\mathrm{CH}_{3} \mathrm{OH}\right)_{n}\left(\mathrm{H}_{2} \mathrm{O}\right), n=1-4$. recorded with the VUV-ionization/IR-depletion technique. J. Chem. Phys. 2017, 146, 144308. [CrossRef]

12. Huisken, F.; Kaloudis, M.; Kulcke, A.; Laush, C.; Lisy, J.M. Vibrational spectroscopy of small $(\mathrm{HF})_{n}$ clusters $(n=4-8)$ in size-selected molecular beams. J. Chem. Phys. 1995, 103, 5366-5377. [CrossRef]

13. Lucas, B.; Lecomte, F.; Reimann, B.; Barth, H.D.; Grégoire, G.; Bouteiller, Y.; Schermann, J.P.; Desfrançois, C. A new infrared spectroscopy technique for structural studies of mass-selected neutral polar complexes without chromophore. Phys. Chem. Chem. Phys. 2004, 6, 2600-2604. [CrossRef]

14. $\mathrm{Hu}, \mathrm{Y} . J . ; \mathrm{Fu}$, H.B.; Bernstein, E.R. Infrared plus vacuum ultraviolet spectroscopy of neutral and ionic methanol monomers and clusters: New experimental results. J. Chem. Phys. 2006, 125, 154306. [CrossRef]

15. León, I.; Montero, R.; Longarte, A.; Fernández, J.A. IR mass-resolved spectroscopy of complexes without chromophore: cyclohexanol. $\left(\mathrm{H}_{2} \mathrm{O}\right)_{n}, n=1-3$ and cyclohexanol dimer. J. Chem. Phys. 2013, 139, 174312. [CrossRef]

16. Yatsyna, V.; Bakker, D.J.; Salén, P.; Feifel, R.; Rijs, A.M.; Zhaunerchyk, V. Infrared Action Spectroscopy of Low-Temperature Neutral Gas-Phase Molecules of Arbitrary Structure. Phys. Rev. Lett. 2016, 117, 118101. [CrossRef]

17. Zhang, B.; Yu, Y.; Zhang, Z.; Zhang, Y.Y.; Jiang, S.; Li, Q.; Yang, S.; Hu, H.S.; Zhang, W.; Dai, D.; et al. Infrared Spectroscopy of Neutral Water Dimer Based on a Tunable Vacuum Ultraviolet Free Electron Laser. J. Phys. Chem. Lett. 2020, 11, 851-855. [CrossRef]

18. Lovejoy, C.M.; Nesbitt, D.J. Slit pulsed valve for generation of long-path-length supersonic expansions. Rev. Sci. Instrum. 1987, 58, 807-811. [CrossRef]

19. Liu, K.; Fellers, R.S.; Viant, M.R.; McLaughlin, R.P.; Brown, M.G.; Saykally, R.J. A long path length pulsed slit valve appropriate for high temperature operation: Infrared spectroscopy of jet-cooled large water clusters and nucleotide bases. Rev. Sci. Instrum. 1996, 67, 410-416. [CrossRef]

20. Amirav, A.; Even, U.; Jortner, J. Absorption spectroscopy of ultracold large molecules in planar supersonic expansions. Chem. Phys. Lett. 1981, 83, 1-4. [CrossRef]

21. Wang, Z.; Eliades, M.; Carron, K.; Bevan, J.W. A cw planar jet computer-controlled tunable IR diode laser spectrometer for the investigation of hydrogen-bonded complexes. Rev. Sci. Instrum. 1991, 62, 21-26. [CrossRef]

22. Asselin, P.; Dupuis, B.; Perchard, J.P.; Soulard, P. The gas phase infrared spectrum of $\mathrm{HCl}$ complexed with dimethyl ether revisited: Assignment of the fundamental transition from a jet-cooled experiment. Chem. Phys. Lett. 1997, 268, 265-272. [CrossRef]

23. Amrein, A.; Quack, M.; Schmitt, U. High-resolution interferometric Fourier transform infrared absorption spectroscopy in supersonic free jet expansions: Carbon monoxide, nitric oxide, methane, ethyne, propyne, and trifluoromethane. J. Phys. Chem. 1988, 92, 5455-5466. [CrossRef]

24. Suhm, M.A.; Kollipost, F. Femtisecond single-mole infrared spectroscopy of molecular clusters. Phys. Chem. Chem. Phys. 2013, 15, 10702-10721. [CrossRef] [PubMed]

25. Lippe, M.; Szczepaniak, U.; Hou, G.L.; Chakrabarty, S.; Ferreiro, J.J.; Chasovskikh, E.; Signorell, R. Infrared Spectroscopy and Mass Spectrometry of $\mathrm{CO}_{2}$ Clusters during Nucleation and Growth. J. Phys. Chem. A 2019, 123, 2426-2437. [CrossRef] [PubMed]

26. Hartz, C.; Wofford, B.A.; Meads, R.F.; Lucchese, R.R.; Bevan, J.W. A near-infrared Fourier-transform planar supersonic jet spectrometer for rovibrational studies of weakly bound dimers and trimers. Rev. Sci. Instrum. 1995, 66, 4375-4384. [CrossRef]

27. Herman, M.; Georges, R.; Hepp, M.; Hurtmans, D. High resolution Fourier transform spectroscopy of jet-cooled molecules. Int. Rev. Phys. Chem. 2000, 19, 277-325. [CrossRef]

28. Heger, M.; Scharge, T.; Suhm, M.A. From hydrogen bond donor to acceptor: The effect of ethanol fluorination on the first solvating water molecule. Phys. Chem. Chem. Phys. 2013, 15, 16065-16073. [CrossRef]

29. Kollipost, F.; Papendorf, K.; Lee, Y.F.; Lee, Y.P.; Suhm, M.A. Alcohol dimers-How much diagonal OH anharmonicity? Phys. Chem. Chem. Phys. 2014, 16, 15948-15956. [CrossRef]

30. Kollipost, F.; Andersen, J.; Mahler, D.W.; Heimdal, J.; Heger, M.; Suhm, M.A.; Wugt Larsen, R. The effect of hydrogen bonding on torsional dynamics: A combined far-infrared jet and matrix isolation study of methanol dimer. J. Chem. Phys. 2014, 141, 174314. [CrossRef]

31. Kollipost, F.; Domanskaya, A.V.; Suhm, M.A. Microscopic Roots of Alcohol-Ketone Demixing: Infrared Spectroscopy of Methanol-Acetone Clusters. J. Phys. Chem. A 2015, 119, 2225-2232. [CrossRef]

32. Medel, R.; Heger, M.; Suhm, M.A. Molecular Docking via Olefinic OH $\cdots \pi$ Interactions: A Bulky Alkene Model System and Its Cooperativity. J. Phys. Chem. A 2015, 119, 1723-1730. [CrossRef] [PubMed]

33. Heger, M.; Altnöder, J.; Poblotzki, A.; Suhm, M.A. To $\pi$ or not to $\pi$-How does methanol dock onto anisole? Phys. Chem. Chem. Phys. 2015, 17, 13045-13052. [CrossRef] [PubMed]

34. Heger, M.; Mata, R.A.; Suhm, M.A. Soft hydrogen bonds to alkenes: The methanol-ethene prototype under experimental and theoretical scrutiny. Chem. Sci. 2015, 6, 3738-3745. [CrossRef]

35. Gottschalk, H.C.; Altnöder, J.; Heger, M.; Suhm, M.A. Control over the Hydrogen-Bond Docking Site in Anisole by Ring Methylation. Angew. Chem. Int. Ed. 2016, 55, 1921-1924. [CrossRef] [PubMed]

36. Kollipost, F.; Otto, K.E.; Suhm, M.A. A Symmetric Recognition Motif between Vicinal Diols: The Fourfold Grip in Ethylene Glycol Dimer. Angew. Chem. Int. Ed. 2016, 55, 4591-4595. [CrossRef] 
37. Medcraft, C.; Zinn, S.; Schnell, M.; Poblotzki, A.; Altnöder, J.; Heger, M.; Suhm, M.A.; Bernhard, D.; Stamm, A.; Dietrich, F.; et al. Aromatic embedding wins over classical hydrogen bonding-A multi-spectroscopic approach for the diphenyl ether-methanol complex. Phys. Chem. Chem. Phys. 2016, 18, 25975-25983. [CrossRef]

38. Poblotzki, A.; Altnöder, J.; Suhm, M.A. Subtle solvation behaviour of a biofuel additive: The methanol complex with 2,5dimethylfuran. Phys. Chem. Chem. Phys. 2016, 18, 27265-27271. [CrossRef]

39. Forsting, T.; Gottschalk, H.C.; Hartwig, B.; Mons, M.; Suhm, M.A. Correcting the record: The dimers and trimers of trans-Nmethylacetamide. Phys. Chem. Chem. Phys. 2017, 19, 10727-10737. [CrossRef]

40. Oswald, S.; Wallrabe, M.; Suhm, M.A. Cooperativity in Alcohol-Nitrogen Complexes: Understanding Cryomatrices through Slit Jet Expansions. J. Phys. Chem. A 2017, 121,3411-3422. [CrossRef]

41. Bernhard, D.; Dietrich, F.; Fatima, M.; Perez, C.; Poblotzki, A.; Jansen, G.; Suhm, M.A.; Schnell, M.; Gerhards, M. Multispectroscopic and theoretical analyses on the diphenyl ether-tert-butyl alcohol complex in the electronic ground and electronically excited state. Phys. Chem. Chem. Phys. 2017, 19, 18076-18088. [CrossRef]

42. Oswald, S.; Suhm, M.A. Experimental Reference Data for Hexafluorinated Propanol by Exploring an Unusual Intermolecular Torsional Balance. Angew. Chem. Int. Ed. 2017, 56, 12672-12676. [CrossRef]

43. Meyer, K.A.E.; Suhm, M.A. Formic acid aggregation in 2D supersonic expansions probed by FTIR imaging. J. Chem. Phys. 2017, 147, 144305. [CrossRef]

44. Poblotzki, A.; Gottschalk, H.C.; Suhm, M.A. Tipping the Scales: Spectroscopic Tools for Intermolecular Energy Balances. J. Phys. Chem. Lett. 2017, 8, 5656-5665. [CrossRef] [PubMed]

45. Gottschalk, H.C.; Poblotzki, A.; Suhm, M.A.; Al-Mogren, M.M.; Antony, J.; Auer, A.A.; Baptista, L.; Benoit, D.M.; Bistoni, G.; Bohle, F.; et al. The furan microsolvation blind challenge for quantum chemical methods: First steps. J. Chem. Phys. 2018, 148, 014301. [CrossRef]

46. Oswald, S.; Meyer, E.; Suhm, M.A. Dinitrogen as a Sensor for Metastable Carboxylic Acid Dimers and a Weak Hydrogen Bond Benchmarking Tool. J. Phys. Chem. A 2018, 122, 2933-2946. [CrossRef] [PubMed]

47. Bernhard, D.; Dietrich, F.; Fatima, M.; Pérez, C.; Gottschalk, H.C.; Wuttke, A.; Mata, R.A.; Suhm, M.A.; Schnell, M.; Gerhards, M. The phenyl vinyl ether-methanol complex: A model system for quantum chemistry benchmarking. Beilstein J. Org. Chem. 2018, 14, 1642-1654. [CrossRef]

48. Meyer, K.A.E.; Suhm, M.A. Vibrational exciton coupling in homo and hetero dimers of carboxylic acids studied by linear infrared and Raman jet spectroscopy. J. Chem. Phys. 2018, 149, 104307. [CrossRef] [PubMed]

49. Oswald, S.; Suhm, M.A.; Coussan, S. Incremental NH stretching downshift through stepwise nitrogen complexation of pyrrole: A combined jet expansion and matrix isolation study. Phys. Chem. Chem. Phys. 2019, 21, 1277-1284. [CrossRef] [PubMed]

50. Oswald, S.; Seifert, N.A.; Bohle, F.; Gawrilow, M.; Grimme, S.; Jäger, W.; Xu, Y.; Suhm, M.A. The Chiral Trimer and a Metastable Chiral Dimer of Achiral Hexafluoroisopropanol: A Multi-Messenger Study. Angew. Chem. Int. Ed. 2019, 58, 5080-5084. [CrossRef]

51. Karir, G.; Lüttschwager, N.O.B.; Suhm, M.A. Phenylacetylene as a gas phase sliding balance for solvating alcohols. Phys. Chem. Chem. Phys. 2019, 21, 7831-7840. [CrossRef] [PubMed]

52. Medel, R.; Stelbrink, C.; Suhm, M.A. Vibrational Signatures of Chirality Recognition Between $\alpha$-Pinene and Alcohols for Theory Benchmarking. Angew. Chem. Int. Ed. 2019, 58, 8177-8181. [CrossRef] [PubMed]

53. Oswald, S.; Suhm, M.A. Soft experimental constraints for soft interactions: A spectroscopic benchmark data set for weak and strong hydrogen bonds. Phys. Chem. Chem. Phys. 2019, 21, 18799-18810. [CrossRef] [PubMed]

54. Zimmermann, C.; Gottschalk, H.C.; Suhm, M.A. Three-dimensional docking of alcohols to ketones: An experimental benchmark based on acetophenone solvation energy balances. Phys. Chem. Chem. Phys. 2020, 22, 2870-2877. [CrossRef]

55. Gottschalk, H.C.; Poblotzki, A.; Fatima, M.; Obenchain, D.A.; Pérez, C.; Antony, J.; Auer, A.A.; Baptista, L.; Benoit, D.M.; Bistoni, G.; et al. The first microsolvation step for furans: New experiments and benchmarking strategies. J. Chem. Phys. 2020, 152, 164303. [CrossRef] [PubMed]

56. Medel, R.; Suhm, M.A. Understanding benzyl alcohol aggregation by chiral modification: The pairing step. Phys. Chem. Chem. Phys. 2020, 22, 25538-25551. [CrossRef] [PubMed]

57. Zimmermann, C.; Fischer, T.L.; Suhm, M.A. Pinacolone-Alcohol Gas-Phase Solvation Balances as Experimental Dispersion Benchmarks. Molecules 2020, 25, 5095. [CrossRef] [PubMed]

58. Nejad, A.; Suhm, M.A.; Meyer, K.A.E. Increasing the weights in the molecular work-out of cis- and trans-formic acid: Extension of the vibrational database via deuteration. Phys. Chem. Chem. Phys. 2020, 22, 25492-25501. [CrossRef] [PubMed]

59. Carney, J.R.; Zwier, T.S. Infrared and Ultraviolet Spectroscopy of Water-Containing Clusters of Indole, 1-Methylindole, and 3-Methylindole. J. Phys. Chem. A 1999, 103, 9943-9957. [CrossRef]

60. Robertson, E.G. IR-UV ion-dip spectroscopy of N-phenyl formamide, and its hydrated clusters. Chem. Phys. Lett. 2000, 325, 299-307. [CrossRef]

61. Robertson, E.G.; Hockridge, M.R.; Jelfs, P.D.; Simons, J.P. IR-UV Ion-Dip Spectroscopy of N-Benzylformamide Clusters: Stepwise Hydration of a Model Peptide. J. Phys. Chem. A 2000, 104, 11714-11724. [CrossRef]

62. Mons, M.; Dimicoli, I.; Tardivel, B.; Piuzzi, F.; Robertson, E.G.; Simons, J.P. Energetics of the Gas Phase Hydrates of transFormanilide: A Microscopic Approach to the Hydration Sites of the Peptide Bond. J. Phys. Chem. A 2001, 105, 969-973. [CrossRef] 
63. Le Barbu, K.; Lahmani, F.; Mons, M.; Broquier, M.; Zehnacker, A. IR-UV investigation of the structure of the 1-phenylethanol chromophore and its hydrated complexes. Phys. Chem. Chem. Phys. 2001, 3, 4684-4688. [CrossRef]

64. Sakai, D.; Matsuda, Y.; Hachiya, M.; Mori, M.; Fujii, A.; Mikami, N. Size-selected Infrared Predissociation Spectroscopy of Neutral and Cationic Formamide-water clusters: Stepwise growth of hydrated structures and intracluster hydrogen transfer induced by vacuum-ultraviolet photoionization. J. Phys. Chem. A 2008, 112, 6840-6849. [CrossRef]

65. Fricke, H.; Schwing, K.; Gerlach, A.; Unterberg, C.; Gerhards, M. Investigations of the water clusters of the protected amino acid Ac-Phe-OMe by applying IR/UV double resonance spectroscopy: Microsolvation of the backbone. Phys. Chem. Chem. Phys. 2010, 12, 3511-3521. [CrossRef]

66. Cirtog, M.; Asselin, P.; Soulard, P.; Tremblay, B.; Madebène, B.; Alikhani, M.E.; Georges, R.; Moudens, A.; Goubet, M.; Huet, T.R.; et al. The $\left(\mathrm{CH}_{2}\right)_{2} \mathrm{O}-\mathrm{H}_{2} \mathrm{O}$ Hydrogen Bonded Complex. Ab Initio Calculations and Fourier Transform Infrared Spectroscopy from Neon Matrix and a New Supersonic Jet Experiment Coupled to the Infrared AILES Beamline of Synchrotron SOLEIL. J. Phys. Chem. A 2011, 115, 2523-2532. [CrossRef] [PubMed]

67. Schwing, K.; Reyheller, C.; Schaly, A.; Kubik, S.; Gerhards, M. Structural Analysis of an Isolated Cyclic Tetrapeptide and its Monohydrate by Combined IR/UV Spectroscopy. ChemPhysChem 2011, 12, 1981-1988. [CrossRef] [PubMed]

68. Zischang, J.; Lee, J.J.; Suhm, M.A. Communication: Where does the first water molecule go in imidazole? J. Chem. Phys. 2011, 135, 061102. [CrossRef] [PubMed]

69. Hepp, M.; Herregodts, F.; Herman, M. Fourier transform infrared jet spectroscopy using a heated slit source. Chem. Phys. Lett. 1998, 294, 528-532. [CrossRef]

70. Okada, Y.; Isomura, S.; Satooka, S.; Takeuchi, K. Pulsed Laval nozzle with a large optical path length of $50 \mathrm{~cm}$. Rev. Sci. Instrum. 1994, 65, 2414-2415. [CrossRef]

71. Potapov, A.; Asselin, P. High-resolution jet spectroscopy of weakly bound binary complexes involving water. Int. Rev. Phys. Chem. 2014, 33, 275-300. [CrossRef]

72. Huisken, F.; Kaloudis, M.; Kulcke, A. Infrared spectroscopy of small size-selected water clusters. J. Chem. Phys. 1996, 104, 17-25. [CrossRef]

73. Mukhopadhyay, A.; Cole, W.T.; Saykally, R.J. The water dimer I: Experimental characterization. Chem. Phys. Lett. 2015, 633, 13-26. [CrossRef]

74. Page, R.H.; Frey, J.G.; Shen, Y.R.; Lee, Y.T. Infrared predissociation spectra of water dimer in a supersonic molecular beam. Chem. Phys. Lett. 1984, 106, 373-376. [CrossRef]

75. Paul, J.B.; Collier, C.P.; Saykally, R.J.; Scherer, J.J.; O’Keefe, A. Direct Measurement of Water Cluster Concentrations by Infrared Cavity Ringdown Laser Absorption Spectroscopy. J. Phys. Chem. A 1997, 101, 5211-5214. [CrossRef]

76. Paul, J.B.; Provencal, R.A.; Chapo, C.; Petterson, A.; Saykally, R.J. Infrared cavity ringdown spectroscopy of water clusters: O-D stretching bands. J. Chem. Phys. 1998, 109, 10201-10206. [CrossRef]

77. General Discussion. Faraday Disc. 2001, 118, 295-314. [CrossRef]

78. Häber, T.; Schmitt, U.; Emmeluth, C.; Suhm, M.A. Ragout-jet FTIR spectroscopy of cluster isomerism and cluster dynamics: From carboxylic acid dimers to $\mathrm{N}_{2} \mathrm{O}$ nanoparticles. Faraday Disc. 2001, 118, 331-359; discussion 361-371. [CrossRef]

79. Moudens, A.; Georges, R.; Goubet, M.; Makarewicz, J.; Lokshtanov, S.E.; Vigasin, A.A. Direct absorption spectroscopy of water clusters formed in a continuous slit nozzle expansion. J. Chem. Phys. 2009, 131, 204312. [CrossRef]

80. Nizkorodov, S.A.; Ziemkiewicz, M.; Nesbitt, D.J.; Knight, A.E.W. Overtone spectroscopy of $\mathrm{H}_{2} \mathrm{O}$ clusters in the $v_{(\mathrm{OH})}=2$ manifold: Infrared-ultraviolet vibrationally mediated dissociation studies. J. Chem. Phys. 2005, 122, 194316. [CrossRef]

81. Tremblay, B.; Bouteiller, Y.; Perchard, J.P. Identification of two quanta simultaneous vibrational transitions in the dimer water trapped in inert matrices. Chem. Phys. 2011, 382, 15-19. [CrossRef]

82. Suas-David, N.; Vanfleteren, T.; Földes, T.; Kassi, S.; Georges, R.; Herman, M. The Water Dimer Investigated in the 2OH Spectral Range Using Cavity Ring-Down Spectroscopy. J. Phys. Chem. A 2015, 119, 10022-10034. [CrossRef] [PubMed]

83. Bhargava, R.; Fernandez, D.C.; Schaeberle, M.D.; Levin, I.W. Theory and Application of Gain Ranging to Fourier Transform Infrared Spectroscopic Imaging. Appl. Spectrosc. 2001, 55, 1580-1589. [CrossRef]

84. Heger, M. Diagonal and Off-Diagonal Anharmonicity in Hydrogen-Bonded Systems. Ph.D. Thesis, Georg-August-Universität Göttingen, Göttingen, Germany, 2015. Available online: https:/ /hdl.handle.net/11858/00-1735-0000-0028-874B-E (accessed on 11 March 2021).

85. Meyer, V.; Eisermann, S.; Gottschalk, H.C.; Hildebrandt, R.; Langer, B.; Schmitt, U.; Zippert, M.; Suhm, M.A. Gratin Jet Figures: Version 1.0. 2020. Available online: https:/ / doi.org/10.25625/6V6KSN (accessed on 11 March 2021). [CrossRef]

86. Gottschalk, H.C. IR-Untersuchung von Schwach Gebundenen Molekülaggregaten im Überschallstrahl. Ph.D. Thesis, GeorgAugust-Universität Göttingen, Göttingen, Germany, 2020. Available online: https://hdl.handle.net/21.11130/00-1735-0000-0005 -14EE-9 (accessed on 11 March 2021).

87. Mertz, L. Auxiliary computation for Fourier spectrometry. Infrared Phys. 1967, 7, 17-23. [CrossRef]

88. Camy-Peyret, C.; Flaud, J.M.; Guelachvili, G.; Amiot, C. High resolution Fourier transform spectrum of water between 2930 and $4255 \mathrm{~cm}^{-1}$. Mol. Phys. 1973, 26, 825-855. [CrossRef]

89. Emmeluth, C.; Dyczmons, V.; Kinzel, T.; Botschwina, P.; Suhm, M.A.; Yáñez, M. Combined jet relaxation and quantum chemical study of the pairing preferences of ethanol. Phys. Chem. Chem. Phys. 2005, 7, 991-997. [CrossRef] [PubMed] 
90. Wassermann, T.N.; Suhm, M.A. Ethanol Monomers and Dimers Revisited: A Raman Study of Conformational Preferences and Argon Nanocoating Effects. J. Phys. Chem. A 2010, 114, 8223-8233. [CrossRef]

91. Fischer, T.L.; Wagner, T.; Gottschalk, H.C.; Nejad, A.; Suhm, M.A. A Rather Universal Vibrational Resonance in 1:1 Hydrates of Carbonyl Compounds. J. Phys. Chem. Lett. 2021, 12, 138-144. [CrossRef]

92. Gottschalk, H.C.; Meyer, V.; Hildebrandt, R.; Schmitt, U.; Fischer, T.L.; Suhm, M.A. Data Repository for Suhm Publication [186] 2021. Available online: https:/ / doi.org/10.25625/CNAP14 (accessed on 11 March 2021).

93. Zhang, B.; Yu, Y.; Zhang, Y.Y.; Jiang, S.; Li, Q.; Hu, H.S.; Li, G.; Zhao, Z.; Wang, C.; Xie, H.; et al. Infrared spectroscopy of neutral water clusters at finite temperature: Evidence for a noncyclic pentamer. Proc. Natl. Acad. Sci. USA 2020, 117, 15423-15428. [CrossRef]

94. Otto, K.E.; Xue, Z.; Zielke, P.; Suhm, M.A. The Raman spectrum of isolated water clusters. Phys. Chem. Chem. Phys. 2014, 16, 9849-9858. [CrossRef]

95. Kuyanov-Prozument, K.; Choi, M.Y.; Vilesov, A.F. Spectrum and infrared intensities of OH-stretching bands of water dimers. J. Chem. Phys. 2010, 132, 014304. [CrossRef]

96. León, I.; Montero, R.; Castaño, F.; Longarte, A.; Fernández, J.A. Mass-Resolved Infrared Spectroscopy of Complexes without Chromophore by Nonresonant Femtosecond Ionization Detection. J. Phys. Chem. A 2012, 116, 6798-6803. [CrossRef]

97. Coker, D.F.; Miller, R.E.; Watts, R.O. The infrared predissociation spectra of water clusters. J. Chem. Phys. 1985, 82, 3554-3562. [CrossRef]

98. Wuelfert, S.; Herren, D.; Leutwyler, S. Supersonic jet CARS spectra of small water clusters. J. Chem. Phys. 1987, 86, 3751-3753. [CrossRef]

99. Lee, J.J.; Höfener, S.; Klopper, W.; Wassermann, T.N.; Suhm, M.A. Origin of the Argon Nanocoating Shift in the OH Stretching Fundamental of $n$-Propanol: A Combined Experimental and Quantum Chemical Study. J. Phys. Chem. C 2009, 113, 10929-10938. [CrossRef]

100. Meyer, K.A.E.; Davies, J.A.; Ellis, A.M. Shifting formic acid dimers into perspective: Vibrational scrutiny in helium nanodroplets. Phys. Chem. Chem. Phys. 2020, 22, 9637-9646. [CrossRef]

101. Michielsen, B.; Dom, J.J.J.; van der Veken, B.J.; Hesse, S.; Xue, Z.; Suhm, M.A.; Herrebout, W.A. The complexes of halothane with benzene: The temperature dependent direction of the complexation shift of the aliphatic $\mathrm{C}-\mathrm{H}$ stretching. Phys. Chem. Chem. Phys. 2010, 12, 14034-14044. [CrossRef] [PubMed]

102. Kjaersgaard, A.; Vogt, E.; Hansen, A.S.; Kjaergaard, H.G. Room Temperature Gas-Phase Detection and Gibbs Energies of Water Amine Bimolecular Complex Formation. J. Phys. Chem. A 2020, 124, 7113-7122. [CrossRef]

103. Herschel, W. XIV. Experiments on the Refrangibility of the invisible Rays of the sun. Phil. Trans. R. Soc. 1800, 90, 284-292. [CrossRef]

104. Forsting, T.; Zischang, J.; Suhm, M.A.; Eckhoff, M.; Schröder, B.; Mata, R.A. Strained hydrogen bonding in imidazole trimer: A combined infrared, Raman, and theory study. Phys. Chem. Chem. Phys. 2019, 21, 5989-5998. [CrossRef]

105. Roy, T.K.; Mani, D.; Schwaab, G.; Havenith, M. A close competition between O-H $\cdots$ O and O-H $\cdots \pi$ hydrogen bonding: IR spectroscopy of anisole-methanol complex in helium nanodroplets. Phys. Chem. Chem. Phys. 2020, 22, 22408-22416. [CrossRef] [PubMed] 